\title{
Diagnostischer Stellenwert der Koronarangiographie mittels Mehrschicht- Computertomographie bei Patienten mit symptomatischem Vorhofflimmern vor Pulmonalvenenablation
}

\author{
INAUGURAL - DISSERTATION \\ zur Erlangung des Doktorgrades \\ der Medizinischen Fakultät der \\ Georg-August-Universität zu Göttingen
}

vorgelegt von

Sebastian Heinz Herbert Kruse

aus

Aachen

Göttingen 2016 
Dekan:

Referent:

Ko-Referent:

Drittreferent:
Prof. Dr. rer. nat. H. K. Kroemer

Prof. Dr. med. M. Zabel

Prof. Dr. med. O. Ritter

Prof. Dr. med. T. Paul

Datum der mündlichen Prüfung: $\quad$ 24.05.2017 


\section{INHALTSVERZEICHNIS}

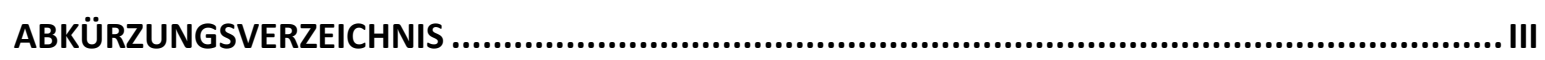

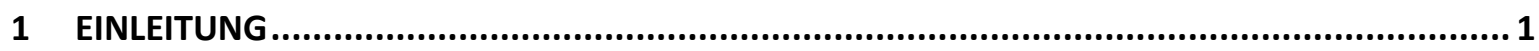

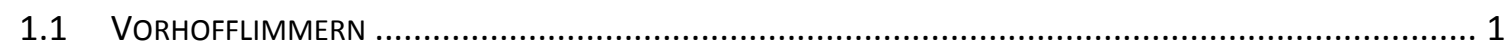

1.1.1 Epidemiologie und Einteilung des Vorhofflimmerns ................................................ 1

1.1.2 Pathophysiologische Hintergründe .............................................................................. 3

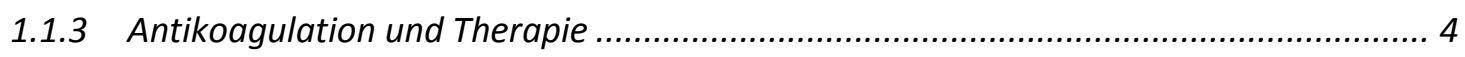

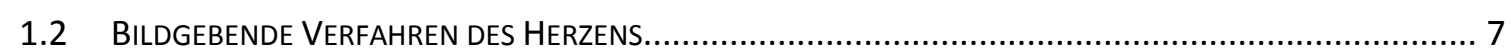

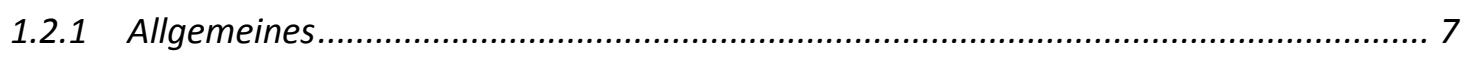

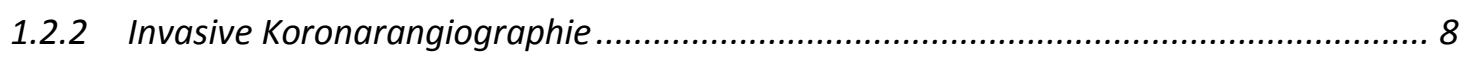

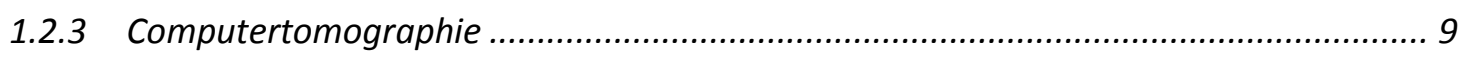

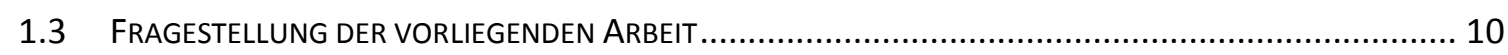

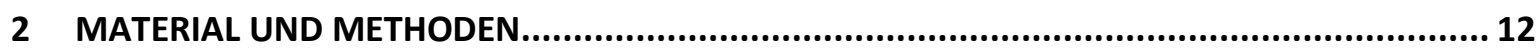

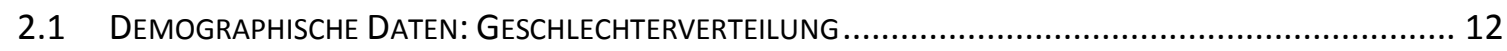

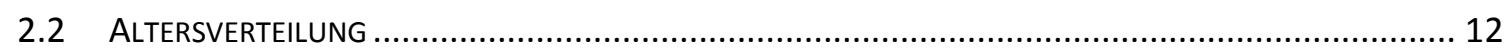

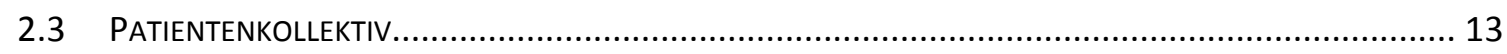

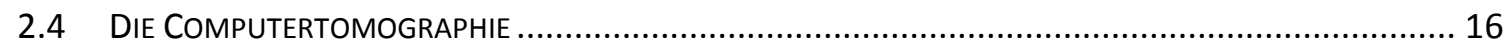

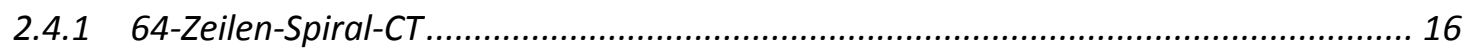

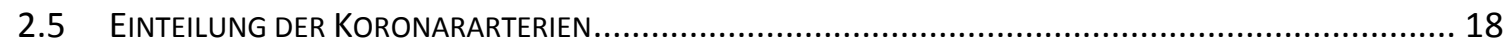

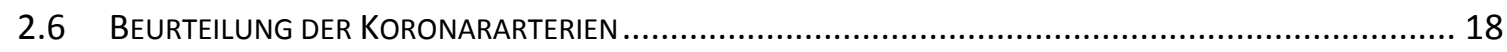

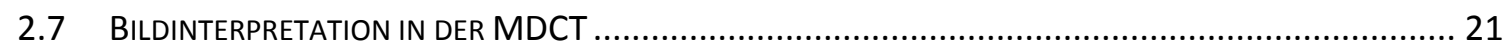

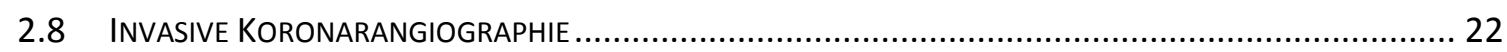

2.8.1 Prozedere der invasiven Koronarangiographie.......................................................... 22

2.8.2 Auswertung der Aufnahmen der invasiven Koronarangiographie ............................. 22

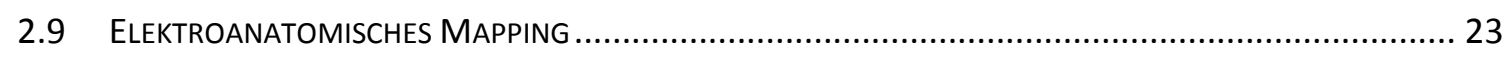

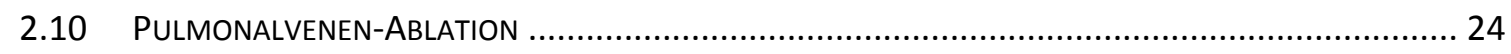

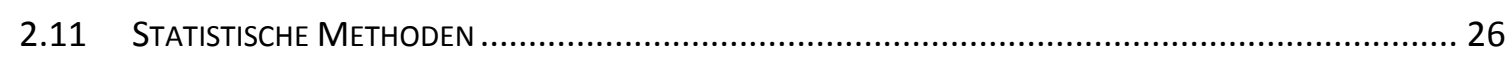

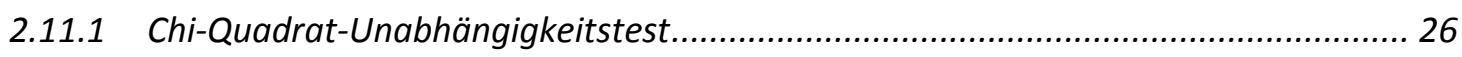

2.11.2 Sensitivität, Spezifität und Vorhersagewert ......................................................... 26

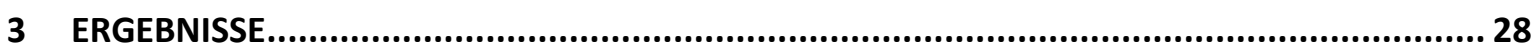




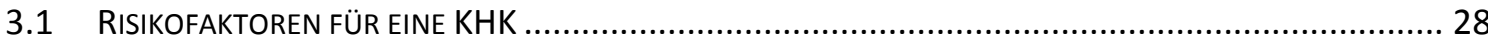

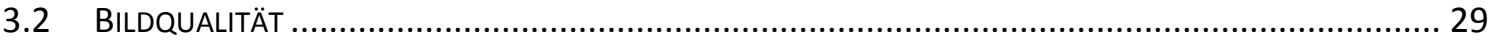

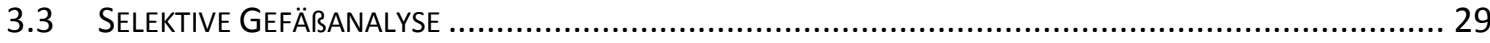

3.3.1 Ergebnisse aller Segmente in der MDCT-Koronarangiographie ................................. 29

3.3.2 Detektion von Stenosen in der invasiven Koronarangiographie ................................ 31

3.4 VERGLEICH VON MDCT-KoRONARANGIOGRAPHIE UND INVASIVER KoRONARANGIOGRAPHIE ............... 31

3.4.1 Unterschiede in der Detektion von Stenosen .............................................................. 33

3.4.2 Sensitivität und Spezifität ................................................................................... 35

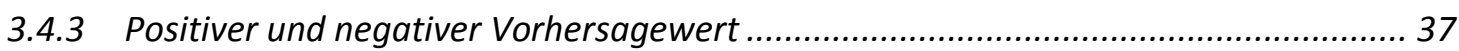

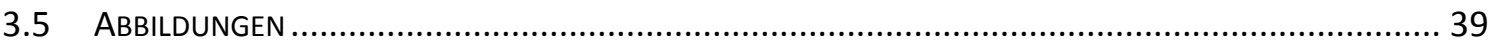

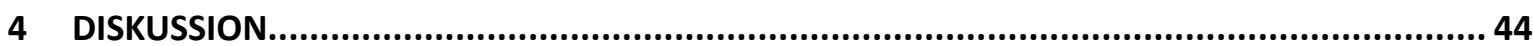

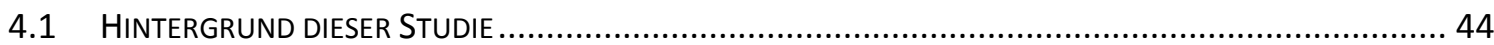

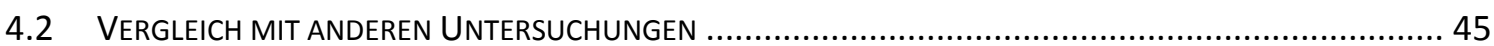

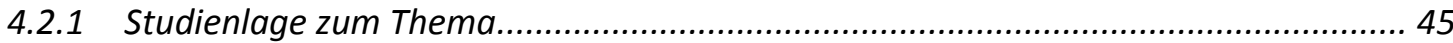

4.2.2 Vergleich der Ergebnisse mit anderen Studien ......................................................... 45

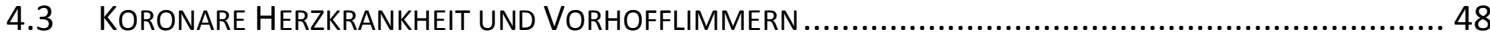

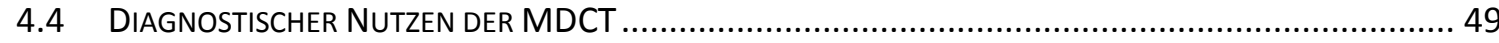

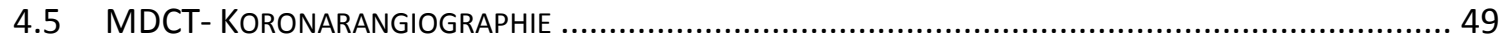

4.5.1 Einflussfaktoren und technische Weiterentwicklung ................................................. 49

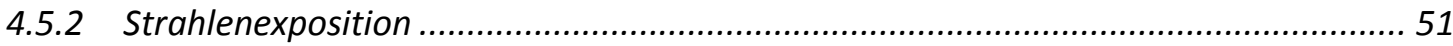

4.5.3 Kosteneffektivität der MDCT-Koronarangiographie im Vergleich zur invasiven

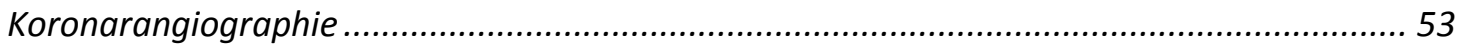

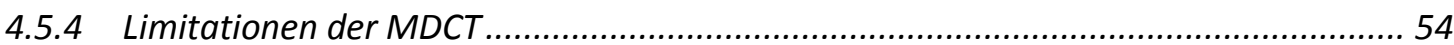

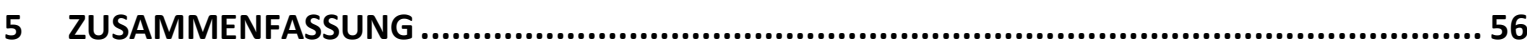

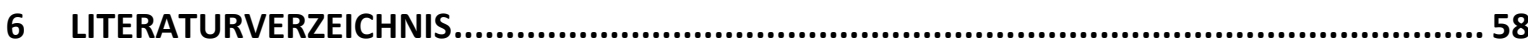




\section{Abkürzungsverzeichnis}

\begin{tabular}{|c|c|}
\hline Abkürzung & Bedeutung \\
\hline ACD & Arteria coronaria dextra \\
\hline ACS & Arteria coronaria sinistra \\
\hline ACT & aktivierte Koagulationszeit \\
\hline $\mathrm{AHA}$ & American Heart Association \\
\hline AKS & akutes Koronarsyndrom \\
\hline AP & Angina pectoris \\
\hline CT & Computertomographie \\
\hline CTA & Computertomographie Angiographie \\
\hline $3 \mathrm{D}$ & dreidimensional \\
\hline 3DM & dreidimensionales Mappingsystem \\
\hline et al. & et alii (und andere) \\
\hline EBM & einheitlicher Bewertungsmaßstab \\
\hline GOÄ & Gebührenordnung für Ärzte \\
\hline HF & Herzfrequenz \\
\hline KHK & koronare Herzerkrankung \\
\hline KM & Kontrastmittel \\
\hline LA & linksatrial \\
\hline MDCT & Multidetektor-Computertomographie \\
\hline MIP & maximum intensity projection \\
\hline MPR & multiplanare Reformatierung \\
\hline MRT & Magnetresonanztomographie \\
\hline NPW & negativ prädiktiver Wert \\
\hline PKI & perkutane Koronarintervention \\
\hline PPW & positiv prädiktiver Wert \\
\hline PV & Pulmonalvenen \\
\hline PVA & Pulmonalvenenablation \\
\hline R. & Ramus \\
\hline $\mathrm{RCX}$ & Ramus circumflexus \\
\hline RIVA & Ramus interventricularis \\
\hline TEE & transösophageale Echokardiographie \\
\hline VHF & Vorhofflimmern \\
\hline VRT & Volume-Rendering-Technik \\
\hline
\end{tabular}




\section{Einleitung}

\subsection{Vorhofflimmern}

\subsubsection{Epidemiologie und Einteilung des Vorhofflimmerns}

Vorhofflimmern (VHF) ist die häufigste Herzrhythmusstörung im Erwachsenenalter. Es wird geschätzt, dass circa 4,5 Millionen Menschen in Europa an dieser Rhythmusstörung leiden (Fuster et al. 2006). Hierbei zeichnet sich ein deutlicher Anstieg der Prävalenz mit zunehmendem Lebensalter ab. Leiden $1 \%$ der über 60-Jährigen unter Vorhofflimmern, so sind es bereits $5 \%$ der über 69-Jährigen und $10 \%$ aller über 80-Jährigen, die diese Rhythmusstörung beklagen (Ohnesorge et al. 2005). In diesem Zusammenhang bestätigt die Rotterdam-Studie, eine europäisch epidemiologische Studie, den Anstieg der Prävalenz in Abhängigkeit vom Alter. Bei den 55-59-Jährigen lag die Prävalenz bei 0,7 \% und stieg auf $17,8 \%$ in der Altersgruppe der über 85-Jährigen (Heeringa et al. 2006). Ein weiterer wichtiger Punkt ist der geschlechtsspezifische Unterschied; dementsprechend leiden Männer etwa 1,5 mal häufiger an VHF als Frauen (Furberg et al. 1994; Benjamin et al. 1994). Das Lebenszeitrisiko mit Mitte 50 an VHF zu erkranken, ist mit $23,8 \%$ bei Männern und mit 22,2 \% bei Frauen beziffert (Heeringa et al. 2006).

VHF kann zu einer Vielzahl von klinischen Symptomen führen. Häufige Symptome sind Tachykardien/Palpitationen (74\%), Schwäche (44\%), Emesis/Vomitus (41\%), Stenokardien (41\%) und Dyspnoe (36\%). Allerdings bemerken $70 \%$ der Patienten keine Symptome und VHF wird z.B. als Zufallsbefund im EKG dokumentiert (Hannink und Laubinger 1982).

VHF kann ätiologisch als primär oder sekundär eingestuft werden. Primäres VHF tritt ohne erkennbare Ursache bzw. Grunderkrankung auf und wird auch als idiopathisches VHF bzw. Ione atrial fibrillation (Brand al. 1985) bezeichnet. Die Prävalenz hierfür liegt bei etwa $10 \%$.

Bei sekundärem VHF unterscheidet man zwischen kardialen und extrakardialen Ursachen. Extrakardial ist in $50 \%$ der Fälle die arterielle Hypertonie als ursächlich zu sehen (Narayan et al. 1997). Der Diabetes mellitus ist ebenfalls mit VHF vergesellschaftet und führt zu einem 1,4-fach erhöhtem Risiko für diese Rhythmusstörung (Benjamin et al. 1994). Weiterhin zu nennen sind sowohl die manifeste Hyperthyreose, bei der ca. 
10-20 \% der Patienten ein VHF entwickeln (Tajiri et al. 1986) sowie die Lungenembolie als auch chronische Lungenerkrankungen. Bei Letzteren wird von einer Prävalenz von bis zu $3 \%$ ausgegangen (Incalzi et al. 1990). Nicht selten ist eine alkoholtoxische Wirkung beschrieben, so liegt das relative Risiko für VHF bei akutem Alkoholexzess - im Sinne eines Holiday Heart-Syndroms - bei 2,3 (Cohen et al. 1988).

Neben diesen extrakardialen Ursachen zeigen sich ätiologisch häufig strukturelle Herzerkrankungen als Auslöser von VHF. So entwickeln $15 \%$ der Patienten mit kongenitalenVitien, hier insbesondere der Vorhofseptumdefekt, diese Herzrhythmusstörung (Tikoff et al. 1968). Bei der Mitralstenose liegt die Prävalenz bei 40 \% (Alpert et al. 1988), bei der rheumatischen Mitralinsuffizienz sogar bei $75 \%$ (Selzer und Katayama 1972). Weiterhin ist die Herzinsuffizienz als eine der bedeutendsten kardialen Ursachen für VHF zu nennen; hier werden Prävalenzen von 10-20 \% angegeben (Morris und Hurst 1980). Die Prävalenz von VHF liegt bei der hypertrophen Kardiomyopathie bei $10 \%$ (McKenna et al. 1981), bei akuter Perikarditis bei 5 \% (Morris und Hurst 1980) und beim akuten Myokardinfarkt bei ca. 9 \% (Eldar et al. 1998).

Die aktuelle Klassifikation des VHF richtet sich nach den ESC Guidelines (Camm et al. 2010) und unterscheidet hierbei konkret fünf zeitliche Muster (Tabelle 01):

Tabelle 01: Klassifikation des VHF nach den ESC Guidelines (Camm et al. 2010).

\begin{tabular}{cc} 
Form des Vorhofflimmerns & $\begin{array}{c}\text { Charakteristika der jeweiligen Form des } \\
\text { Vorhofflimmerns }\end{array}$ \\
\hline erstmals diagnostiziertes VHF & $\begin{array}{c}\text { Erste Manifestation von VHF, unabhängig } \\
\text { von der Dauer }\end{array}$ \\
persistierendes VHF & $\begin{array}{c}\text { Sistiert spontan }<7 \text { Tage, meist innerhalb } \\
\text { von 48 Stunden }\end{array}$ \\
lang anhaltend persistierendes VHF & Dauer $>7$ Tage oder Beendigung durch \\
Arzt
\end{tabular}




\subsubsection{Pathophysiologische Hintergründe}

Betrachtet man die elektrophysiologischen Hintergründe des VHF, so lassen sich im Wesentlichen zwei ursächliche Mechanismen benennen. Wie in der Studie von Haissaguerre gezeigt, sind atriale Extrasystolen und hochfrequente atriale Tachykardien, im Sinne einer sogenannten Triggerarrhythmie, für die Induktion des Flimmerns verantwortlich (Haissaguerre et al. 1998). Weiterführende Studien anderer Forschungsgruppen deuteten diesbezüglich auf den posterioren linken Vorhof im Übergangsbereich zur Einmündung der Pulmonalvenen (PV) als Ort der Initiierung und Aufrechterhaltung der Rhythmusstörung hin (Sueda et al. 1996; Skanes et al. 1998). Die o.g. Triggerfoci können grundsätzlich in allen vier PV lokalisiert sein, häufig kommen sie jedoch in der linken und rechten Superioren PV vor (Haissagurerre et al. 1998). Pathogenetisch ist das in die PV einstrahlende Vorhofgewebe mit myokardialen Faserbündeln als Ursprung der Foci zu sehen (Narayan et al. 2008).

Ein zweiter Mechanismus sind die multiplen, zirkulierenden atrialen Erregungskreisläufe, welche das arrhythmieerhaltende Flimmersubstrat darstellen und bereits 1959 von Moe und Abildskov mit ihrer multiple-wavelet-Hypothese demonstriert wurde (Moe und Abildskov 1959). Diese besagt, dass VHF aus vielfachen und unregelmäßigen ReentryErregungsfronten entsteht, von denen sich ständig neue Erregungsfronten (daughterwavelets) abspalten. Mittels biatrialer Mappingstudien konnte die Relevanz dieser multiple-wavelet-Hypothese für das VHF verifiziert werden (Cox et al. 1991 a). 


\subsubsection{Antikoagulation und Therapie}

Bei VHF ist, gemäß den aktuellen Leitlinien eine adäquate Antikoagulation zur Prophylaxe eines thromboembolischen Ereignisses notwendig (Camm et al. 2012). Die Risikoabschätzung eines Schlaganfalles bei VHF erfolgt diesbezüglich mit Hilfe des $\mathrm{CHA}_{2} \mathrm{DS}_{2}$-VASc-Scores (Tabelle 02).

Tabelle 02: Erläuterung zum $\mathrm{CHA}_{2} \mathrm{DS}_{2}$-VASc-Score (Camm et al. 2012).

\begin{tabular}{|c|c|c|}
\hline Buchstabe & Bedeutung & Punktzahl \\
\hline C (congestive heart failure) & $\begin{array}{c}\text { strukturelle } \\
\text { Herzerkrankung, die eine } \\
\text { Herzinsuffizienz verursacht }\end{array}$ & 1 \\
\hline $\mathrm{H}$ (hypertension) & $\begin{array}{l}\text { Arterielle Hypertonie } \\
\text { (auch behandelt) }\end{array}$ & 1 \\
\hline $\mathrm{A}_{2}$ (age) & Alter $>75$ Jahre & 2 \\
\hline D (diabetes) & Diabetes mellitus & 1 \\
\hline $\mathrm{S}_{2}$ (stroke) & $\begin{array}{c}\text { durchgemachter } \\
\text { Schlaganfall oder } \\
\text { transitorische ischämische } \\
\text { Attacke }\end{array}$ & 2 \\
\hline V (vascular disease) & $\begin{array}{l}\text { z.B. durchgemachter } \\
\text { Herzinfarkt, pAVK }\end{array}$ & 1 \\
\hline A (age) & Alter 65-74 & 1 \\
\hline $\mathrm{S}(\operatorname{sex})$ & weibliches Geschlecht & 1 \\
\hline
\end{tabular}


In Abhängigkeit von der erreichten Punktzahl und des damit objektivierten Risikoprofils des Patienten, erfolgt keine antithrombotische Therapie (0 Risikofaktoren) oder aber eine orale Antikoagulation mit Phenprocoumon oder Faktor-Xa-Inhibitoren ( $\geq 1$ Risikofaktoren) (Camm et al. 2012; Ru San et al. 2012).

Bei der Therapie des VHF müssen zwei Verfahren unterschieden werden. Zum einen gibt es die Möglichkeit der Frequenzkontrolle, bei der das VHF belassen und in erster Linie die AV-Knoten Erregungsüberleitung bzw. die ventrikuläre Frequenz reguliert werden. Erreicht wird dieses pharmakologisch z.B. mit Betablockern, Digitalispräparaten sowie Calciumantagonisten (Fuster et al. 2006).

Zum anderen kann die Rhythmuskontrolle angestrebt werden, womit gemeint ist, den Sinusrhythmus wiederherzustellen und dauerhaft zu stabilisieren. Hierzu gibt es derzeit mehrere, teils stufenweise angelegte Konzepte:

1. Nach vierwöchiger effektiver Antikoagulation (INR von 2-3) oder nach Ausschluss intrakardialer Thromben mittels TEE erfolgt eine elektrische oder medikamentöse Kardioversion in den Sinusrhythmus.

1.1. Bei der elektrischen Kardioversion erfolgt mittels externem Defibrillator eine elektrische Impulsabgabe, welche unter EKG-Kontrolle mit dem QRS- Komplex synchronisiert wird.

1.2. Bei der medikamentösen Kardioversion stehen grundsätzlich Antiarrhythmika der Klasse IA, IC sowie der Klasse III zur Verfügung. Diese Form der Kardioversion ist innerhalb der ersten 7 Tage nach Auftreten des VHF am effektivsten, anschließend ist eine deutlich rückläufige Erfolgsquote zu verzeichnen (Camm et al. 2010).

2. Als weitere Möglichkeit steht die kathetergestützte Ablationstherapie als Methode der Rhythmuskontrolle zur Verfügung, bei der in den überwiegenden Fällen mittels Hochfrequenzstrom eine zirkumferentielle oder segmentale Pulmonalvenenisolation durchgeführt wird.

3. Weiterhin gibt es aktuell die Möglichkeit der chirurgischen Rhythmuskontrolle. Dies lässt sich bei Patienten mit medikamentös oder interventionell nicht therapierbarem VHF in Betracht ziehen. Historisch dient die klassische Maze-Operation, die von James Cox et al. Ende der 1980er entwickelt wurde und bei der die Vorhöfe zur Isolation mit einem Skalpell inzidiert (maze [engl.] = Irrgarten) und vernäht wurden, als Grundlage. Hierdurch sollten Reentry-Kreise beziehungsweise auslösende elektrische Triggerpunkte 
unterbrochen werden (Cox et al. 1991 b; Khargi et al. 2005; Knaut et al. 2004; Knaut et al. 2007).

Aktuell besteht durch die Weiterentwicklung alternativer Energieformen (Kryothermie, Radiofrequenz, Mikrowelle, Ultraschall, Laser) die Möglichkeit, das oben genannte Narbengewebe ohne die klassische Schnitt- und Nahttechnik zu erzeugen. Diese chirurgische Therapie des VHF war in der Vergangenheit, aufgrund ihrer Invasivität, lediglich als Ergänzung zu einem aus anderen Gründen notwendigen kardiochirurgischen Eingriff (z.B. Bypass-Operation, Herzklappenersatz) sinnvoll (Patwardhan et al. 1997; Fuster et al. 2006). Durch die Weiterentwicklung moderner Ablationssonden ist aktuell jedoch ein thorakoskopisch minimalinvasiver chirurgischer Eingriff möglich, ohne dass die Eröffnung des Thorax notwendig wird (Koistinen et al. 2013).

Swartz et al. starteten in den 90er Jahren erstmals den Versuch einer katheterbasierten Durchführung des Konzeptes von Cox (Swartz et al. 1994). Die technische Möglichkeit zur Durchführung langer linksatrialer Ablationslinien wurde von weiteren Gruppen postuliert (Jais et al. 1999; Ernst et al. 1999). Aufgrund hoher Raten an Komplikationen und einer sehr langen Untersuchungsprozedur wurde dieses Verfahren zunächst als klinisch nicht relevant erachtet. Erst mit Beschreibung der PV als VHF-induzierende Foci und der Möglichkeit der erfolgreichen Katheterablation durch Haissaguerre und Mitarbeiter, rückte dieses Verfahren wieder in den Fokus (Haissaguerre et al. 1994, Haissaguerre et al. 1998). Die Tatsache, dass andere Gruppen ähnliche Ergebnisse präsentierten (Chen et al. 1999; Pappone et al. 2000; Lin et al. 2000; Gerstenfeld et al. 2001), etablierte diese Methode als klinische Therapieoption der Wahl bei antiarrhythmika-refraktärem VHF. Da die Radiofrequenzablation innerhalb der PV jedoch die Gefahr der Stenose mit konsekutiver pulmonaler Hypertonie in sich barg, entwickelten sich wie folgt zwei modifizierte Verfahren: Zum einen die sogenannte segmental-ostiale Pulmonalvenen-Ablation (PVA), bei der die in die PV einstrahlenden Muskelbündel abladiert werden (Haissaguerre et al. 2000 a; Haissaguerre et al. 2000 b; Arentz et al. 2003; Saad et al. 2003). Die Limitation dieser Methode ist darin begründet, dass es neben einer häufigen Erholung des Gewebes und daher erforderlicher Zweitintervention, zu einer durch die fokale Ablation unzureichenden Elimination der Arrhythmie induzierenden Foci im Übergangsbereich der Lungenvenen in den linken Vorhof kommen kann (Hindricks und Kottkamp 2001). Die Erfolgsquote dieser Prozedur wird mit $>80 \%$ beziffert (Oral et al. 2002 b; Arentz et al. 2003).

Zum anderen die zirkumferentielle PVA, bei der zirkumferentielle Läsionen um die PV im Bereich des Antrums gesetzt werden. Unterstützt wird dieses Verfahren durch ein elektroanatomisches Mapping-System, welches die linksatriale Anatomie dreidimensional 
(3D) darstellt, indem auf präprozedurale 3D-Datensätze (MDCT oder MRT) zurückgegriffen wird. Dieser Ansatz, der ebenfalls von der Abteilung Kardiologie und Pneumologie der UMG angewendet wird und erstmals von Pappone 1999 vorgestellt wurde, erreicht Erfolgsraten von 50-85 \%. Durch mehrmalige Ablationen können jedoch auch Erfolgsraten von bis zu 95 \% erreicht werden (Pappone et al. 1999; Pappone et al. 2000; Arentz et al. 2003; Saad et al. 2003).

\subsection{Bildgebende Verfahren des Herzens}

\subsubsection{Allgemeines}

Zum besseren Verständnis der linksatrialen (LA) Anatomie wird heutzutage in vielen Kliniken, vor Durchführung einer Katheterablation eine 3D-Darstellung des Herzens vorgenommen. Dies ist besonders wichtig, da die Anatomie der PV eine essentielle Rolle spielt und deren Anzahl, Durchmesser, Anatomie sowie Mündung wichtig für die Orientierung während der Ablationsbehandlung sind. In diesem Zusammenhang weisen hierbei ca. $40 \%$ der Patienten eine anatomische Variante auf (Mantovan et al. 2005; Sohns et al. 2013).

Des Weiteren wird die 3D-Darstellung auch für das elektroanatomische Mapping im Rahmen der PVA angewendet. So gelingt durch Fusion, der mit dem Mapping-Katheter durch Abtasten der endokardialen Strukturen erstellten elektroanatomischen Landkarte sowie den zuvor schnittbilddiagnostisch gewonnenen Bilddateien eine detailgetreue Darstellung der Anatomie; dies gegebenfalls samt Ortung des Arrhythmieursprunges.

Als diagnostische Methode zur Visualisierung der Herzanatomie kommen hierbei prinzipiell die Magnetresonanztomographie (MRT) als auch die MultidetektorComputertomographie (MDCT) in Frage. Letztere, welche sich als häufiges Verfahren durchgesetzt hat bietet den Vorteil, dass sie nicht nur kostengünstiger ist sondern auch eine kürzere Untersuchungsdauer beinhaltet. Weiterhin können, neben der Darstellung der LA-Anatomie, auch die hämodynamisch relevanten Abschnitte der Koronararterien besser dargestellt werden (Pouleur et al. 2008; Gerber 2009; Sohns 2011, Dorenkamp et al. 2012). Ebenfalls konnte gezeigt werden, dass mittels MDCT mit hoher Sensitivität intrakranielle Thromben nachgewiesen werden können und diese Untersuchung somit 
tendenziell eine Alternative zu der präprozeduralen transösophagealen Echokardiographie (TEE) darstellen kann (Martinez et al. 2009).

Besteht nun bei Patienten, wie bei einem Teil unseres Kollektivs der Fall, neben VHF der Verdacht einer koronaren Herzerkrankung (KHK), so wird dieser heutzutage mittels invasiver Koronarangiographie verifiziert bzw. falsifiziert. In mehreren Studien konnte gezeigt werden, dass bei Verdacht auf eine KHK oder auch dem Vorliegen einer manifesten KHK mittels MDCT eine gute Beurteilung der hämodynamisch relevanten Abschnitte der Koronararterien möglich ist und ein Nachweis stenosierender Prozesse gelingt (Nieman et al. 2002 b; Rubinshtein et al. 2007; Stein et al. 2008).

Die Tatsache, dass die Symptome des VHF oft unspezifisch sind und denen eines akuten Koronarsyndroms (AKS) ähneln können bzw. eine KHK eine Ursache für das Auftreten von VHF sein kann (Eldar et al. 1998) wirft die Frage auf, ob nun auch bei Patienten mit VHF eine adäquate Beurteilung der Koronararterien mittels Routine-MDCT vor geplanter Ablation möglich ist und somit eine invasive Koronarangiographie ersetzten kann.

\subsubsection{Invasive Koronarangiographie}

Bei der invasiven Koronarangiographie stehen als Gefäßzugänge die A. femoralis, die A. brachialis sowie die A. radialis zur Verfügung. Nachdem das entsprechende Gefäß in Seldinger-Technik punktiert wird, erfolgt zur Minimierung des Blutverlustes und zum atraumatischen Einführen und Wechseln der Katheter das Einlegen einer Schleuse in das gewählte Gefäß. Anschließend wird ein Katheter über einen weichen J-förmig gebogenen Führungsdraht unter Röntgendurchleuchtung bis in den Aortenbogen oberhalb der Aortenklappe vorgeschoben. Von hier aus können nun die Koronararterien dargestellt und ihre Lokalisation, Länge und der Schweregrad sowie die Art der Obstruktion erfasst werden. Darüber hinaus ermöglicht dieser Zugangsweg, neben der reinen Diagnostik, ebenfalls eine Intervention im Sinne einer Ballon- bzw. Stentimplantation durchzuführen (Becker et al. 2000).

Neben diesem klaren Vorteil darf man jedoch etwaige Risiken, die eine invasive Diagnostik in sich birgt nicht vernachlässigen. Hierbei nimmt das Risiko mit zunehmendem Alter sowie Ausmaß der Erkrankung zu. So ist die Gesamtmortalität mit $0,11 \%$ beziffert. Neben z.B. peripheren Komplikationen wie AV-Fisteln, Aneurysmata und 
Hämatomen, sind auch ernste Komplikationen wie Infarkte, Kammerflimmern, Lungenödem und Asystolie zu berücksichtigen (Bestehorn 2001).

Vor dem Hintergrund der heutigen Diskussion um stetig steigende Kosten im Gesundheitssystem sind die Kosten für eine invasive Koronarangiographie als weiterer wichtiger Faktor zu erwähnen. Dem einheitlichen Bewertungsmaßstab (EBM) und der Gebührenordnung für Ärzte (GOÄ) zufolge liegen die Kosten hierfür bei 356 Euro (De Haen C 1995; Trümper 2006).

\subsubsection{Computertomographie}

Erwies es sich zu Beginn der 80er Jahre mangels zeitlicher und örtlicher Auflösung als nicht realisierbar, die Koronararterien mittels Computertomographie (CT) darzustellen (Kalender 1999), etablierte sich unter ständiger Weiterentwicklung der CT-Technik 1994 erstmals ein CT mit zwei simultanen Detektorzeilen und 1998 schließlich ein 4-zeiliger MDCT. Durch diese Weiterentwicklung ergab sich eine rasche Verbesserung der diagnostischen Qualität und erstmalig die Möglichkeit, Engstellen der Koronarien zu detektieren. Achenbach et al. präsentierten Ergebnisse, denen zu Folge Koronarstenosen mittels 4-Zeilen-CT bereits mit einer Sensitivität von 58-86 \% evaluiert wurden. Dennoch konnten $32 \%$ der Herzkranzgefäße aufgrund zu schlechter Bildqualität nicht beurteilt werden. Ursächlich wurden hierfür Bildartefakte und Gefäßkalcifikationen benannt. Somit war es selbst unter Einsatz dieser Geräte nicht möglich, verengte Gefäßabschnitte in einer der Koronarangiographie ähnlichen Qualität darzustellen (Achenbach et al. 2001).

Mittels 16-Schicht-CT-Geräten (2001) konnten nun erstmals mit hoher diagnostischer Sicherheit (positiv und negativ prädiktiver Wert: $79 \%$ und $97 \%$ ) signifikante Stenosen ausgeschlossen werden. Doch auch hierbei fanden noch $12 \%$ der Bilder wegen zu artefaktreicher Darstellung keine Verwendung in dem Protokoll (Ropers et al. 2003). Dieser negative Aspekt wurde mit dem 64-Zeilen-CT weiter minimiert. So ist es nicht verwunderlich, dass aktuelle Studien davon ausgehen, signifikante Stenosen mit einer Spezifität von 97-100 \% ausschließen zu können (Leschka et al. 2005; Meijboom et al. 2006; Stein et al. 2008; Sibley und Bluemeke 2009).

Damit war der Höhepunkt der Entwicklung jedoch noch nicht erreicht. Im Folgenden kam es zu einer weiteren Zunahme der Schichtzahlen bis auf dato 640 (320 Zeilen, doppelt ausgelesen) gleichzeitig akquirierbarer Bildzeilen. Des Weiteren wurde 2005 ein DualSource-CT entwickelt, bei der zwei rotierende Röntgenstrahler verwendet werden, die um 
90 Grad versetzt positioniert sind und die mit unterschiedlicher Spannung betrieben werden können. Durch den simultanen Einsatz zweier Röntgenstrahler kann die Aufzeichnungszeit halbiert und, noch wichtiger, die Strahlendosis reduziert werden.

Anfangs verhinderten Tachykardien bzw. tachykarde Arrhythmien, aufgrund der hierdurch entstehenden Bildartefakte, die Beurteilung der Koronararterien und galten somit als Kontraindikation für die Durchführung einer MDCT (Ropers et al. 2003). Ropers et al. konnten jedoch in ihrer Studie zeigen, dass durch die Weiterentwicklung der CT-Technik eine Beurteilung sowohl bei höheren Herzfrequenzen als auch bei Arrhythmien möglich ist (Ropers et al. 2003). Die mit der Weiterentwicklung der CT einhergehenden Möglichkeiten der Visualisierung der Koronararterien verlangt nach einer Vergleichbarkeit mit der invasiven Koronarangiographie als aktueller Goldstandard. Dies führt uns zur Fragestellung der vorliegenden Arbeit.

\subsection{Fragestellung der vorliegenden Arbeit}

Ziel dieser Arbeit war es, den derzeitigen Stellenwert der 64-Zeilen-MDCT zur Beurteilung von hämodynamisch signifikanten Koronarstenosen bei denjenigen Patienten zu evaluieren, die aufgrund eines therapierefraktären, symptomatischen VHF eine Katheterablation erhalten sollten. Als Referenzmethode diente hierbei die konventionelle, invasive Koronarangiographie.

Die Tatsache, dass die KHK, neben Herzklappenvitien, mit 30-60 \% die häufigste kardiale Ursache des VHF bei alten Menschen darstellt (Blomstrom et al. 2003), lässt ein besonderes Augenmerk auf die nichtinvasive Detektion von Stenosen legen. Durch die zügige technologische Weiterentwicklung der CT, verbunden mit ihrer immer besser werdenden zeitlichen und räumlichen Auflösung, ist die Bildgebung des Herzens mittels CT aktueller denn je. Viele Studien setzten sich mit der derzeitigen CT-Angiographie (CTA) auseinander, wobei sie viel diskutiert, gewürdigt und auch kritisch hinterfragt wird. Genau aus diesem Grund soll im Folgenden dargelegt werden, wie gut die Evaluierung der Koronararterien - hierbei vor allem die hämodynamisch relevanten Gefäßabschnitte - bei Patienten mit symptomatischem VHF im CT gelingt. Dies ist besonders vor dem Hintergrund interessant, dass bei unserem Patientenkollektiv, zur Planung der PVA, eine vorausgehende MDCT notwendig ist und mit einer Untersuchung zwei diagnostische Schritte zusammengeführt werden. Somit hypothetisierten wir, dass 
bei Patienten mit VHF in der präprozeduralen Routine-MDCT vor Ablation, nichtinvasiv und ohne erneute Strahlenbelastung, eine Aussage über etwaige Stenosen der Koronararterien getroffen werden kann. 


\section{Material und Methoden}

\subsection{Demographische Daten: Geschlechterverteilung}

Von den in die Studie eingeschlossenen 181 Patienten waren 124 Männer (69 \%) und 57 (31\%) Frauen. Hieraus ergibt sich ein männlich/weiblich-Verhältnis von 2,2/1 (Abb. 01).

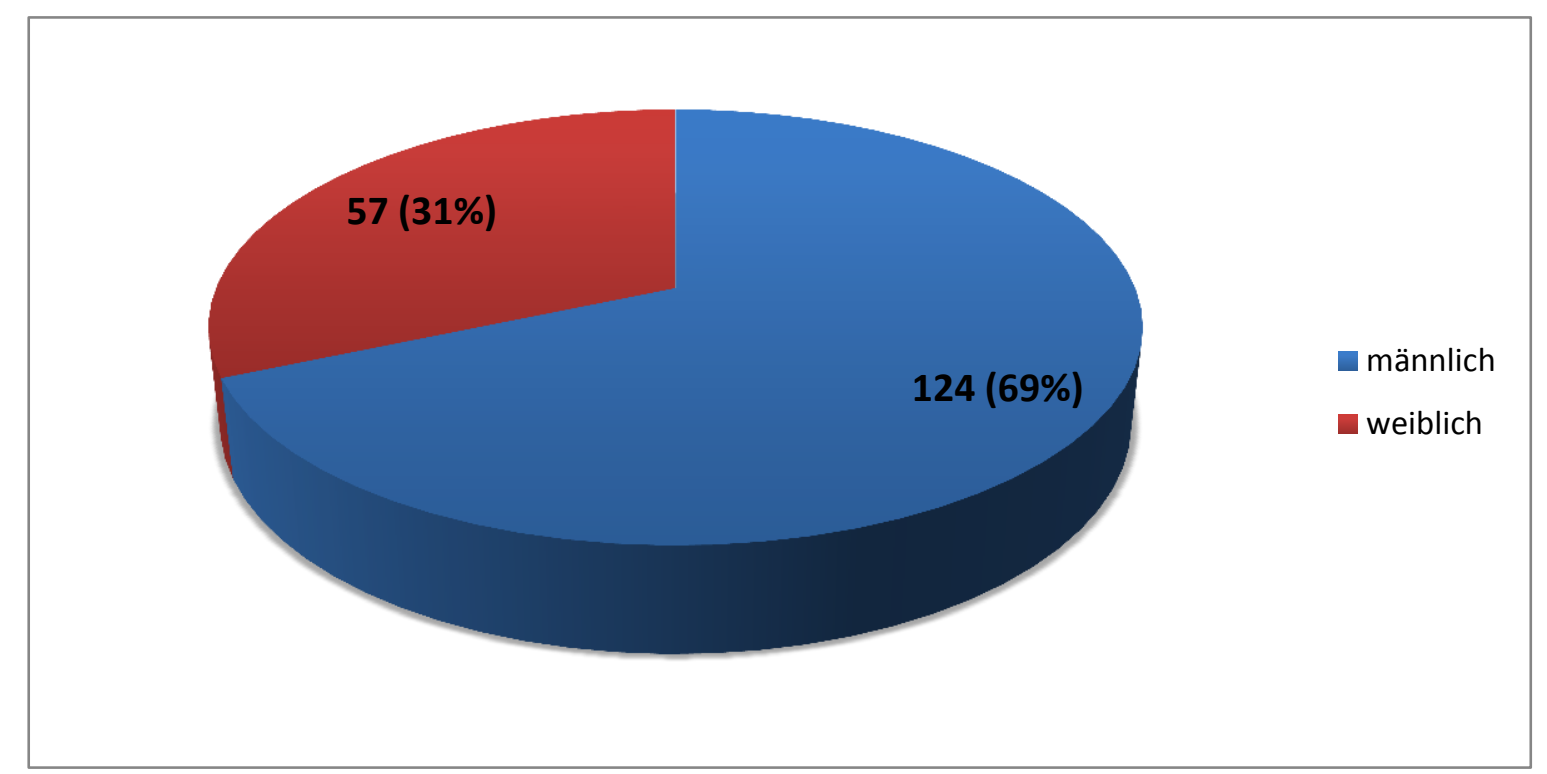

Abbildung 01: Verteilung der Patienten nach Geschlecht $(\mathrm{n}=181)$.

\subsection{Altersverteilung}

Zum Zeitpunkt der Studie war der jüngste Patient 21 Jahre alt, der älteste erfasste Patient war 82 Jahre alt. Im Durchschnitt betrug das Alter der Patienten 57 Jahre mit einer Standardabweichung von 11 Jahren. Folgendes Schaubild demonstriert die Altersverteilung innerhalb der Studie (Abb. 02): 


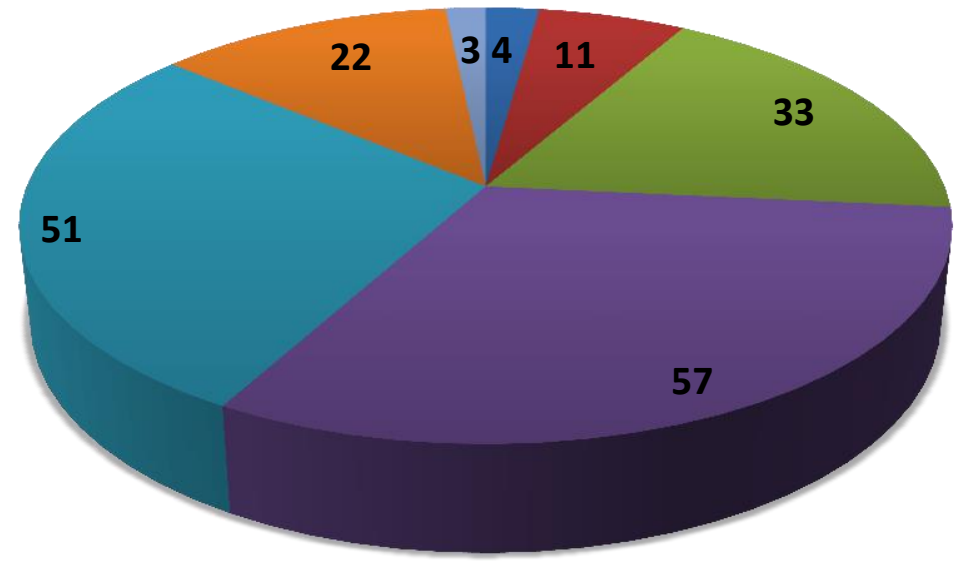

- 20-29 Jahre

- 30-39 Jahre

40-49 Jahre

50-59 Jahre

60-69 Jahre

70-79 Jahre

80-89 Jahre

Abbildung 02: Verteilung der Patienten nach Alter $(n=181)$.

\subsection{Patientenkollektiv}

Alle Patienten dieser Arbeit litten unter symptomatischem therapierefraktärem VHF. Nach dem Scheitern eines medikamentösen Therapieversuches war somit bei dem gesamten Patientenkollektiv eine PVA indiziert. Im Vorfeld der kathetergeführten Ablationsbehandlung des VHF wurde bei allen Patienten eine MDCT durchgeführt, um die hierbei gewonnenen Daten u.a. während der Ablation als 3D-Datensatz im Rahmen des elektroanatomischen Mappings zu verwenden. Stellte sich klinisch der Verdacht einer KHK, wurde im Anschluss an die CT-Untersuchung und vor der PVA eine invasive Koronarangiographie durchgeführt. Die Befunde der MDCT und der invasiven Koronarangiographie wurden jeweils unabhängig von einander, ohne Kenntnis der jeweilig anderen Ergebnisse ausgewertet.

Das Patientenkollektiv wurde in der Göttinger Universitätsklinik - Abteilung Kardiologie und Pneumologie - rekrutiert, untersucht und therapiert. Patienten-Charakteristika sowie Einschlusskriterien sind in Tabelle 03 zusammengefasst.

Als Prognoseinstrument zur Risikostratifizierung des individuellen Gesamtrisikos für kardiovaskuläre Ereignisse existieren mehrere Scoresysteme. In unserer Studie wurde diesbezüglich initial mittels Duke Clinical Score die Wahrscheinlichkeit für eine 
Koronarstenose bewertet. Dieses an der Duke-Universität in North Carolina entwickelte Score-System beinhaltet Art der Thoraxbeschwerden, Alter, Geschlecht sowie folgende Risikofaktoren: Nikotinkonsum (in den letzten 5 Jahren), Gesamtcholesterin, Diabetes mellitus, vorangegangener Herzinfarkt, Q-Zacken oder persistierende ST-Streckenveränderungen im EKG (Pryor et al. 1993; Gibbons et al. 2002). Abhängig vom jeweiligen Ergebnis wurde folgend entschieden, ob neben der MDCT bzw. der PVA eine invasive Koronarangiographie durchgeführt werden musste.

Zusammenfassend konnte bei unserem Patientenkollektiv nach obigem Schema zwischen einer niedrigen ( $1 \%$ bis $30 \%$ ), einer mittleren (31\% bis $70 \%$ ) und einer hohen (71\% bis $99 \%$ ) Wahrscheinlichkeit für das Vorliegen einer signifikanten Koronarstenose unterschieden werden. Folglich wurden bei 86 (48 \%) Patienten mit mittlerem Risiko jeweils eine MDCT und eine invasive Koronarangiographie durchgeführt. Dagegen erhielten 95 (52\%) Patienten, die keine Angina pectoris (AP) Beschwerden oder APÄquivalente sowie im Score ein niedriges Risiko aufwiesen lediglich eine MDCT ohne Durchführung einer invasiven Koronarangiographie (Abb. 03).

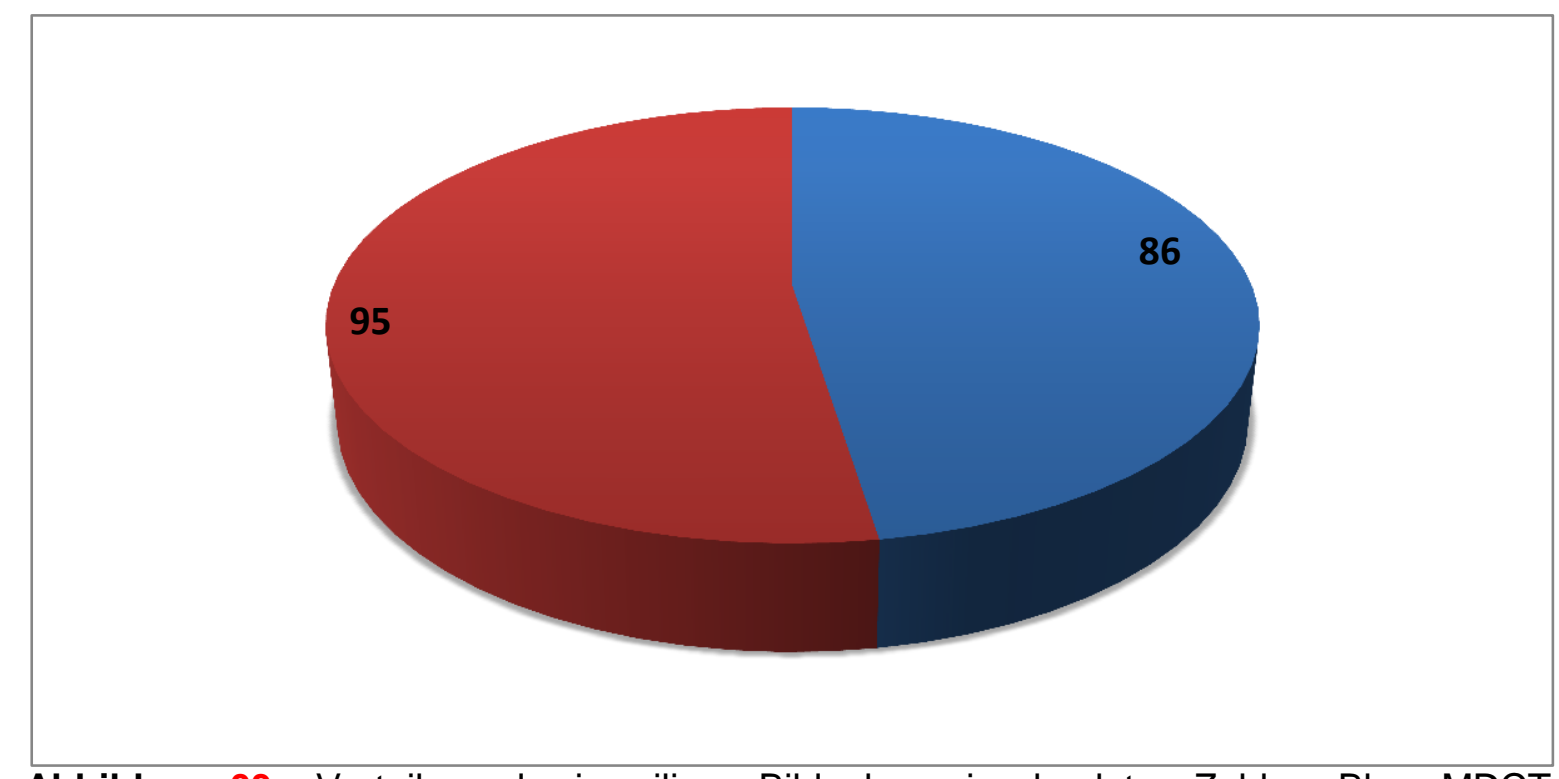

Abbildung 03: Verteilung der jeweiligen Bildgebung in absoluten Zahlen. Blau: MDCT und invasive Koronarangiographie. Rot: Invasive Koronarangiographie. 
Tabelle 03: Charakteristika der 181 Patienten mit symptomatischem VHF.

\begin{tabular}{|c|c|}
\hline Patienten Charakteristika & $\mathrm{n}=181$ \\
\hline Anzahl der Patienten & 181 \\
\hline $\begin{array}{l}\text { Patienten mit invasiver Koronarangiographie und MDCT } \\
\qquad(\mathrm{n} / \%)\end{array}$ & $86(48)$ \\
\hline Patienten mit MDCT (n/\%) & $95(52)$ \\
\hline Geschlecht männlich/weiblich (n) & $124 / 57$ \\
\hline Alter (Jahre) & $57 \pm 11$ \\
\hline durchschnittliche Herzfrequenz in Ruhe (S/min) & $84 \pm 23$ \\
\hline durchschnittliche Herzfrequenz während der MDCT (S/min) & $67 \pm 8$ \\
\hline Body-Maß-Index $\left(\mathrm{kg} / \mathrm{m}^{2}\right)$ & $27,2 \pm 3,5$ \\
\hline Arterieller Hochdruck (n/\%) & $102(55)$ \\
\hline Diabetes mellitus ( $\mathrm{n} / \%)$ & $9(5)$ \\
\hline Adipositas (n/\%) & $57(31)$ \\
\hline Nikotinkonsum (n/\%) & $16(9)$ \\
\hline Hyperlipidämie (n/\%) & $35(19)$ \\
\hline Duke clinical score ${ }^{1}$ - niedriges Risiko (n/\%) & $95(52)$ \\
\hline Duke clinical score ${ }^{1}$ - mittleres Risiko (n/\%) & $86(47)$ \\
\hline paroxysmales VHF (n/\%) & $102(55)$ \\
\hline persistierendes VHF (n/\%) & $61(33)$ \\
\hline permanentes VHF (n/\%) & $18(10)$ \\
\hline vorherige antiarrhythmische Medikation: Flecainid (n/\%) & $91(50)$ \\
\hline vorherige antiarrhythmische Medikation: Propafenon (n/\%) & $20(11)$ \\
\hline vorherige antiarrhythmische Medikation: Sotalol (n/\%) & $24(13)$ \\
\hline vorherige antiarrhythmische Medikation: Amiodaron (n/\%) & $67(36)$ \\
\hline
\end{tabular}

1 Duke Clinical Score beinhaltet: Patientendetails, Art der Brustschmerzen sowie klinische Risikofaktoren für das Vorliegen einer KHK (Gibbons et al. 2002). 


\subsection{Die Computertomographie}

\subsubsection{4-Zeilen-Spiral-CT}

Damit ein komplikationsloser Ablauf der CT-Untersuchung gewährleistet war, wurden im Vorfeld folgende Ausschlusskriterien festgelegt: eine stattgehabte allergische Reaktion auf Kontrastmittel (KM), eine bestehende Niereninsuffizienz oder allgemein ein KreatininWert im Serum von > 1,5 mg/dl, eine hämodynamische Instabilität, eine instabile AP oder ein akuter Myokardinfarkt. Des Weiteren mussten die Patienten für die Untersuchung im Computertomographen Atembefehle befolgen können.

Alle der Arbeit zugrundeliegenden MDCT-Untersuchungen wurden mit Hilfe eines 64Zeilen-Spiral-CTs (VCT LightSpeed der Firma GE Healthcare, Milwaukee, WI, USA) (Abb. 04) angefertigt. Die Patienten dieser Studie erhielten eine standardisierte CT des Thorax und des Oberbauches mit intravenöser Injektion von Kontrastmittel (Imeron 350, Bracco Imaging Deutschland, Konstanz). Die Bildgebung erfolgte 24-48 Stunden vor geplanter PVA. Bei 110 Patienten (61 \%) lag während der Untersuchung VHF vor. Da die Herzfrequenz (HF) der Patienten ein entscheidender Parameter für die Güte der Bildqualität ist (Schroeder et al. 2002), erhielten $87 \%(n=158)$ der Patienten mit einer HF $>70 \mathrm{~S} / \mathrm{min}$ einen Betablocker.

Die durchschnittliche HF während der MDCT lag bei $67 \pm 8 \mathrm{~S} / \mathrm{min}$. In allen Fällen erhielten die Patienten über eine in der Kubitalvene platzierte Verweilkanüle intravenös $80 \mathrm{ml} \mathrm{KM}$. Die CT Aufnahmen wurden während einer einzelnen Atemanhaltephase angefertigt. Der Scan wurde bei einer Röhrenspannung von 120 kV, einem Röhrenstrom von 155 mAs und einer Kollimation von $64 \quad x \quad 0,625 \quad \mathrm{~mm}$ durchgeführt. Die Gantry-Rotationszeit lag bei 333 ms. Für ein optimales Timing und homogene maximale arterielle Kontrastierung erfolgte ein semi-automatisches Bolus-Tracking, die Bildakquisition erfolgte somit zeitverzögert nach Anflutung des KMs in der Aorta ascendens. Anschließend wurden die Bilddaten als sogenannte Rohdaten auf der Gerätefestplatte gesichert und unter Zuhilfenahme des retrospektiven EKG-Gatings rekonstruiert. 


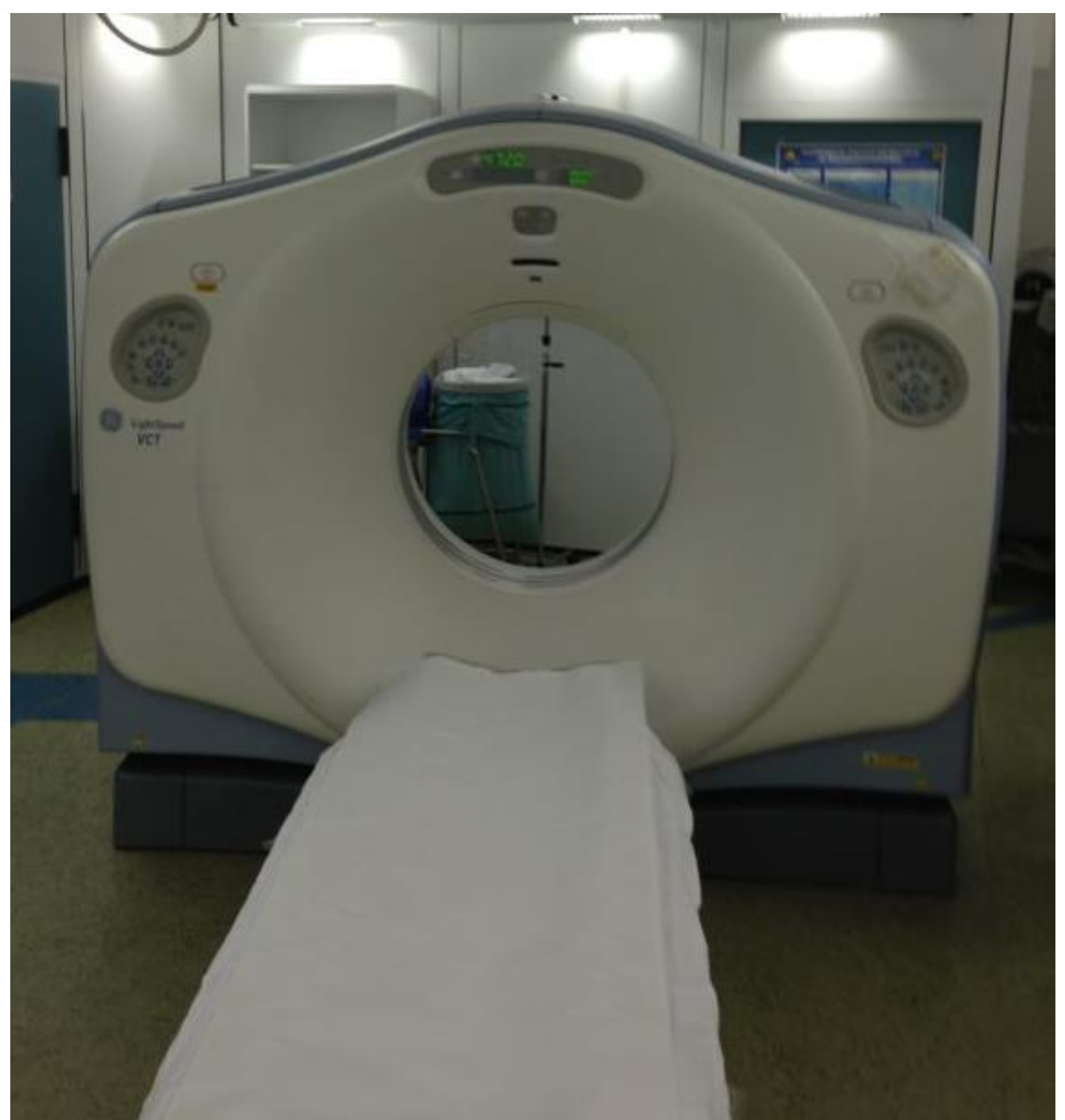

Abbildung 04: 64-Zeilen-Spiral-CT (VCT LightSpeed der Firma GE Healthcare, Milwaukee, WI, USA). 


\subsection{Einteilung der Koronararterien}

Basierend auf einer Einteilung der American Heart Association (AHA) wurden die Koronararterien in 15 Segmente unterteilt (Austen et al. 1975). Die ersten 4 Segmente bezeichnen die rechte Koronararterie (ACD). Das Segment 5 steht für den linken Hauptstamm (ACS). Der linke vordere Ast (RIVA) wird durch die Segmente 6-10 definiert. Die Segmente 11-15 stellen den zirkumflexen Ast dar (RCX) (Abb. 05).

\subsection{Beurteilung der Koronararterien}

Als signifikant wurden bei der Auswertung Stenosen von > $50 \%$ gewertet (Dewey et al. 2006). Stenosen mit < 50 \% Lumeneinengung wurden demnach als nicht hämodynamisch relevant eingestuft, aber dennoch in die Beurteilung einbezogen. Bei mehr als einer Stenose galt die höchstgradige als Referenz. Die Analyse des Koronarbaums erfolgte in allen Gefäßabschnitten mit einem Durchmesser von $\geq 1,5 \mathrm{~mm}$. Die detaillierte Aufteilung gestaltet sich wie folgt (Abb. 05): 


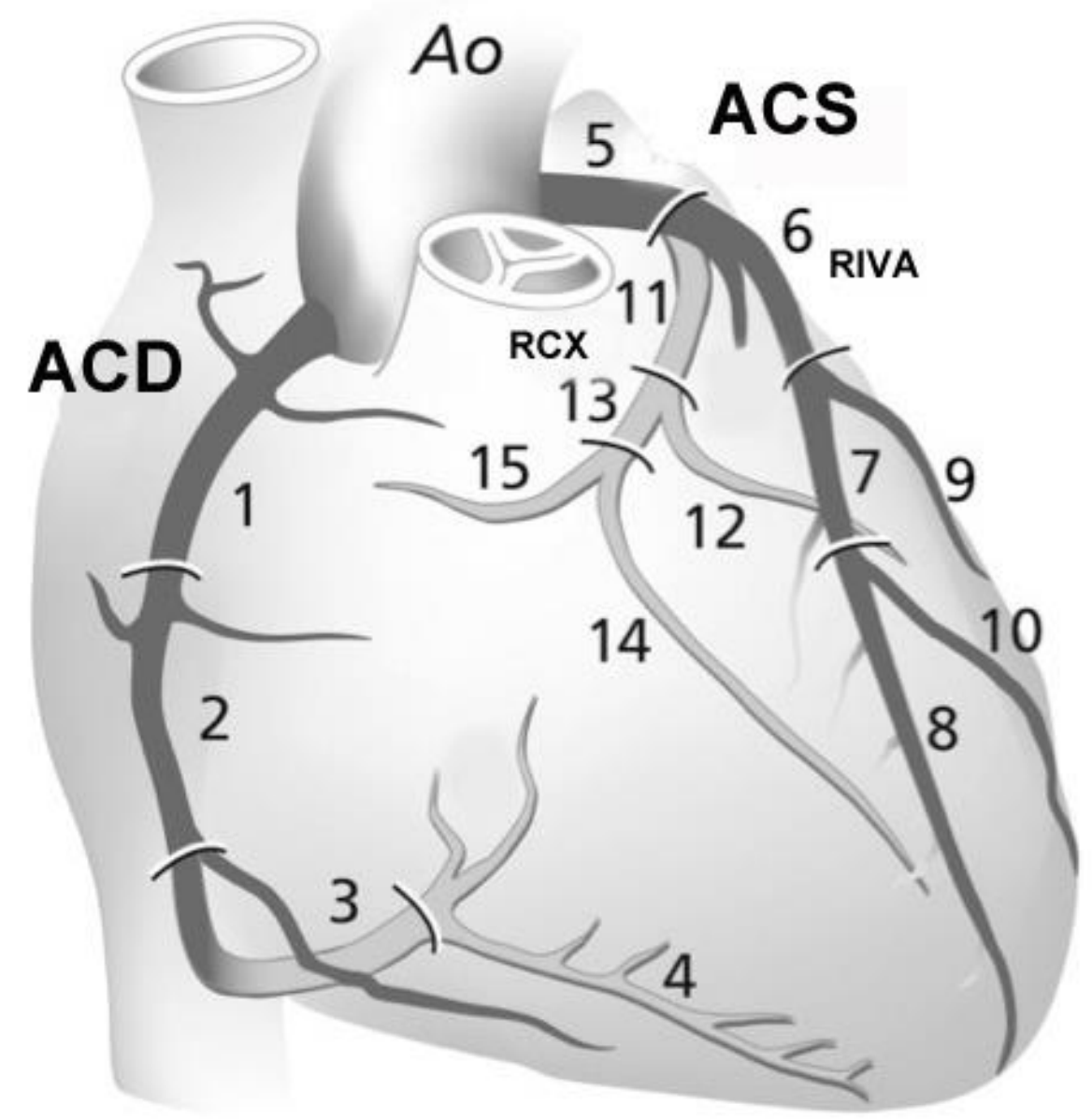

Abbildung 05: Segmentierung der Koronararterien nach der American Heart Association (Austen et al. 1975). Ao = Aorta; $A C D=$ Arteria coronaria dextra; $A C S=$ Arteria coronaria sinistra; RIVA = Ramus interventricularis anterior; RCX = Ramus circumflexus. 
Tabelle 04: Segmentierung der rechten Koronararterie nach AHA.

$\begin{array}{cc}\begin{array}{c}\text { Segmentnummerierung der rechten } \\ \text { Koronararterie }\end{array} & \begin{array}{c}\text { Segmentbezeichung der rechten } \\ \text { Koronararterie }\end{array} \\ 1 & \text { proximaler Abschnitt } \\ 2 & \text { medialer Abschnitt } \\ 3 & \text { distaler Abschnitt } \\ 4 & \text { Ramus interventricularis posterior }\end{array}$

Tabelle 05: Segmentierung der linken Koronararterie nach AHA.

\section{Segmentnummerierung der linken Segmentbezeichnung der linken \\ Koronararterie Koronararterie}

5

6

7

8

9

10

11

12

13

14

15
Linker Hauptstamm (ACS)

R. interventricularis (RIVA): proximaler Abschnitt

R. interventricularis (RIVA): medialer Abschnitt

R. interventricularis (RIVA): distaler Abschnitt

1. Diagonalast

2. Diagonalast

R. circumflexus (RCX): proximaler Abschnitt

1. Marginaler Ast

R. circumflexus (RCX): distaler Abschnitt

2. Marginaler Ast

3. Marginaler Ast 


\subsection{Bildinterpretation in der MDCT}

Zur Beurteilung und Nachbearbeitung der Bilder wurde die Advantage Windows Workstation (Volume Share 2, General Electric, Milwaukee, WI) verwendet. Wie oben erwähnt, erfolgte die Einteilung der Koronararterien entsprechend den Leitlinien der AHA (Austen et al. 1975). Wie auch in anderen Publikationen (Schroeder et al. 2002) wurden die Äste der linken Koronararterie [AHA-Segment 9 (Diagonalast 1), 10 (Diagonalast 2), 14 (Marginalast 2) und 15 (Marginalast 3)] sowie das am weitesten distal gelegene Segment der rechten Koronararterie [AHA-Segment 4 (Ramus interventricularis posterior)] aufgrund ihrer kleinen Durchmesser, der Variabilität ihrer Ursprünge und der niedrigen hämodynamischen Relevanz nicht in die selektive Gefäßanalyse mit eingeschlossen.

Die Analyse der Koronararterien erfolgte segmentbezogen. Ihre Bildqualität im Sinne einer diagnostischen Beurteilbarkeit galt dann als ausreichend, sobald zum einen das Gefäß in seiner ganzen Länge, zum anderen das Gefäß in seinem ganzen Durchmesser beurteilbar war (Delhaye et al. 2007; Hamoir et al. 2005; Zhang et al. 2005). Die Interpretation der gewonnenen Bilddaten erfolgte ohne Kenntnis der Ergebnisse der invasiven Koronarangiographie.

In Abhängigkeit von der Anatomie und der Bildqualität des jeweiligen Koronarsegmentes wurden axiale Schichten, multiplanare Reformatierungen (MPR), Maximumintensitätsprojektionen (MIP, engl. maximum intensity projection) sowie 3D-Rekonstruktionen (VRT, engl. Volume-Rendering-Technik) erstellt. Zur weiteren Bearbeitung wurden Knochen-, Lungen- und Weichteilfenster gewählt.

Für die spätere PVA wurden 3D-MDCT-Datensätze des linken Vorhofes und der PV generiert. Die entsprechende Rekonstruktion der Bilder und Integration dieser im Rahmen des elektroanatomischen Mappings erfolgte schließlich an einem separaten Arbeitsplatz (CARTO Merge, Biosense Webster, Diamond Bar, CA, USA3). 


\subsection{Invasive Koronarangiographie}

\subsubsection{Prozedere der invasiven Koronarangiographie}

Die invasive Koronarangiographie erfolgte entsprechend der Standardtechnik des Herzkatheterlabors der Universitätsmedizin Göttingen. Initial wurde in Lokalanästhesie mittels perkutaner Seldinger-Technik die Arteria femoralis communis nach Judkins punktiert. Ein an der Spitze flexibler, röntgendichter Metalldraht wurde danach über die liegende Punktionsnadel in die Arterie vorgeschoben. Nach Entfernung der Punktionsnadel wurde eine flexible 4-6-French Kunststoff-Schleuse mit blutdichtem Ventil in das Gefäß eingeführt. Über diese erfolgte im Anschluss, unter ständiger Röntgenkontrolle das Vorschieben eines Führungsdrahtes bis in die Aorta ascendens. Über diesen Führungsdraht wiederum wurde ein Führungskatheter vorgeschoben und vor die Ostien der Koronarien platziert. Nach Vorschub des Katheters in die Ostien wurde schließlich das nicht-ionische KM Imeron 350 (Bracco Imaging Deutschland, Konstanz) manuell freigesetzt. Für die biplanen Aufnahmen wurde das Zwei-Röhren-System Integris H 3000 (Philips, Eindhoven, Niederlande) verwendet.

Parallel zu dieser Diagnostik wurden der invasive Blutdruck und das EKG aufgezeichnet. Stellte sich die Indikation zur Intervention, wurde die Stenose nach Wahl eines passenden Führungskatheters mittels Ballonkatheter aufgedehnt. Die Entscheidung zur anschließenden Stentimplantation wurde individuell getroffen.

\subsubsection{Auswertung der Aufnahmen der invasiven Koronarangiographie}

Die invasive Koronarangiographie diente bei unserem Patientenkollektiv als Goldstandard und Vergleichsmethode zur MDCT, die Angiographien wurden von einem erfahrenen Kardiologen analysiert. Wie bei den CT-Aufnahmen, diente auch bei der Analyse der invasiven Koronarangiographie die Segmenteinteilung der Koronarien nach der AHA als Grundlage (Austen et al. 1975). 


\subsection{Elektroanatomisches Mapping}

Dreidimensionale farblich kodierte Mappingsysteme (3DM) des Herzens sind entscheidend für die katheterinterventionelle Therapie, da sie durch die Darstellung des Ursprunges sowie der Ausbreitung einer Arrhythmie mögliche Ablationsziele identifizieren und darüber hinaus, in den Händen erfahrener Untersucher Durchleuchtungs- und Prozedurdauer reduzieren können (Rotter et al. 2005).

Eine erfolgreiche Anwendung eines 3DM setzt die exakte Erstellung eines 3D-Maps voraus, welches aus einer Fusion von hochauflösender Bildgebung wie MRT oder CT und 3D-Elektroanatomie entsteht (CartoMergeXP, Biosense Webster). Das Mapping mit dem Carto-System beruht auf elektromagnetischen Prinzipien. Hierzu werden unter dem Untersuchungstisch drei Magnetspulen angeordnet, die drei unterschiedliche magnetische Wechselfelder generieren. Mittels Bewegung der in das Herz eingebrachten Diagnostikoder Ablationskatheter, die über einen in der Spitze liegenden elektroanatomischen Sensor verfügen, können nun die extrakorporal aufgebauten magnetischen Felder erfasst werden und mittels mathematischer Algorithmen Echtzeitinformationen über die Lokalisation der Katheterspitzen berechnet werden. Dies geschieht mit einer Genauigkeit von $<1 \mathrm{~mm}$. Durch Abtasten der endokardialen Kontur der entsprechenden Herzhöhle kann so eine virtuelle 3D-Rekonstruktion erfolgen. In Verbindung mit der bei Wandkontakt simultan erfassten intrakardialen lokalen Potentiale, erfolgt zusätzlich die Registrierung der Erregungsabläufe der jeweiligen Herzhöhlen bzw. die genaue Darstellung des Verlaufes der Arrhythmie. Über den zeitlichen Abstand zwischen dem Potential des jeweiligen Punktes in einem Map und der Erregung in einer Referenzableitung kann nun die Ausbreitung einer Erregungsfront in einem sogenannten Aktivierungsmap farbkodiert dargestellt werden. In Form einer Animation kann diese Erregungsausbreitung in einem propagation map sichtbar gemacht werden.

Narbenareale oder Areale mit verminderter Potentialgröße, wie beispielsweise infarziertes Myokardgewebe, werden durch lokale Erfassung der Potentialgröße im Voltage-Map erfasst (Gepstein et al. 1997; Willem et al. 2000).

Zur weiteren Optimierung der Darstellung der kardialen Strukturen können nun die im Vorfeld angefertigten MDCT-Aufnahmen des linken Vorhofes und der PV mit den Daten des elektroanatomischen Mappings fusioniert werden. Dies erlaubt eine präzise 3DAbbildung des linken Vorhofes und der PV sowie die exakte räumliche Orientierung und Steuerung der Katheter während der Ablation. Des Weiteren kann so das durch die Kathetermanipulation in real time erzeugte elektroanatomische Bild (MAP) durch die CTBilder validiert und ergänzt werden (Morillo et al. 1995). 


\subsection{Pulmonalvenen-Ablation}

Die PV-Ablation erfolgte unter Analgosedierung und Lokalanästhesie mit intravenös appliziertem Midazolam, Sufentanyl sowie fakultativ Propofol. Anschließend wurden, mittels zweimaliger Punktion der rechten V. femoralis und Einführen zweier Führungsdrähte venöse Schleusen gelegt. Nach Seldinger-Technik erfolgte das Vorschieben eines zehnpoligen steuerbaren 6-French-Katheters (Bard Dynamic TipTM, Bard Inc., Lowell, MA, USA) über die Vena femoralis bis in den Koronarvenensinus.

Eine zweite Schleuse (SL1, St. Jude Medical, Inc., St. Paul, Minnesota, USA) wurde zunächst in den rechten Vorhof vorgebracht. Über diese Schleuse wurde dann die transseptale Punktionsnadel in den rechten Vorhof geführt. Danach erfolgte unter Röntgenkontrolle das Aufsuchen der Fossa ovalis und an dieser Stelle die transseptale Punktion vom rechten in den linken Vorhof. Anschließend wurde die SL1 Schleuse in den linken Vorhof vorgeschoben. Über diese wurde der MAP-Katheter in dem linken Vorhof plaziert. Für die Ablation wurde entweder ein manuell gesteuerter (3,5mm NaviStar ${ }^{\circledR}$ ThermoCool $\AA$, Biosense Webster) oder magnetisch steuerbarer (3,5mm NaviStar ${ }^{\circledR}$ ThermoCool® RMT, Biosense Webster, Diamond Bar, USA) Mapping- und Ablationskatheter verwendet. Im Anschluss an die Punktion des Vorhofseptums erfolgte eine sofortige Vollheparinisierung, die unter Kontrolle der aktivierten Koagulationszeit (ACT) mit einem Zielbereich von 250 und 300 Sekunden während der gesamten Untersuchungsdauer fortgeführt wurde.

Mit Hilfe des MAP-Katheters wurde zunächst, durch sequentielles Abtasten der endokardialen Oberfläche ein virtuelles 3D elektroanatomisches Mapping des linken Vorhofes und der PV (Carto Merge XP, Biosense Webster) erstellt und dieses im Anschluss mit den zuvor angefertigten MDCT-Aufnahmen fusioniert (Abb. 06). Nach Anfertigung des elektroanatomischen Mappings, wurden mit dem Ablationskatheter durch gezielte Applikation von Radiofrequenzstrom kontinuierliche zirkuläre Läsionen im PVAntrum appliziert. Hierbei wurden jeweils beide PV einer Seite in eine solche zirkuläre Läsion eingeschlossen (Abb. 07).

Die Steuerung des Katheters während der Untersuchung erfolgte entweder manuell oder beim magnetischen Verfahren über einen Joystick (CardiodriveTM, Stereotaxis) und das magnetische Navigationssystem Niobe® II (Stereotaxis).

Erzeugt wurde die Radiofrequenzenergie von einem Radiofrequenzgenerator (Stockert, Biosense Webster) mit einer maximalen Katheter-Temperatur von $45^{\circ} \mathrm{C}$ und einer maximalen Energie von $40 \mathrm{~W}$ (Fluss $30 \mathrm{ml} / \mathrm{min}$ ) sowie $30 \mathrm{~W}$ (Fluss $17 \mathrm{ml} / \mathrm{min}$ ) im Bereich der Hinterwand des linken Vorhofes. Die Applikationsdauer auf das Endokard der Wand 
des linken Atriums lag jeweils bei 30 bis 60 Sekunden, bis eine Reduktion der Amplitudengröße im Elektrogramm um mindestens 80 \% zu verzeichnen war. Endpunkt der Ablation war die vollständige Isolation der Pulmonalvenen, also das Fehlen jeglicher elektrischer Leitung zwischen dem linken Vorhof und den PV.

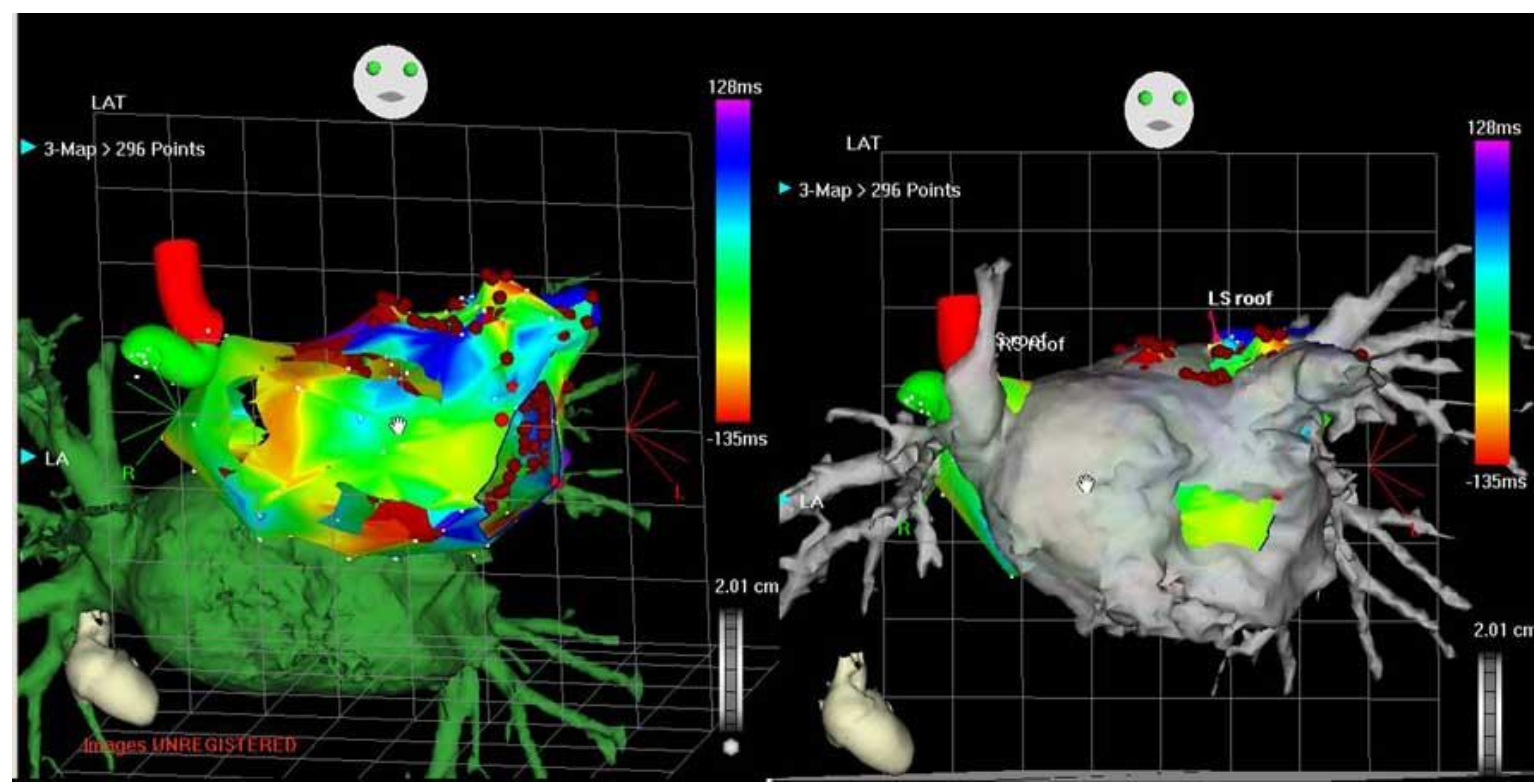

Abbildung 06: Links mit grün dargestellt ist die CT rekonstruierte Anatomie des linken Vorhofes, bunt dargestellt ist das kathetergestützt erstellte Modell. Rechts ist die Fusion beider Modelle erfolgt (CT-Anatomie jetzt grau) (Deisenhofer et al. 2006).

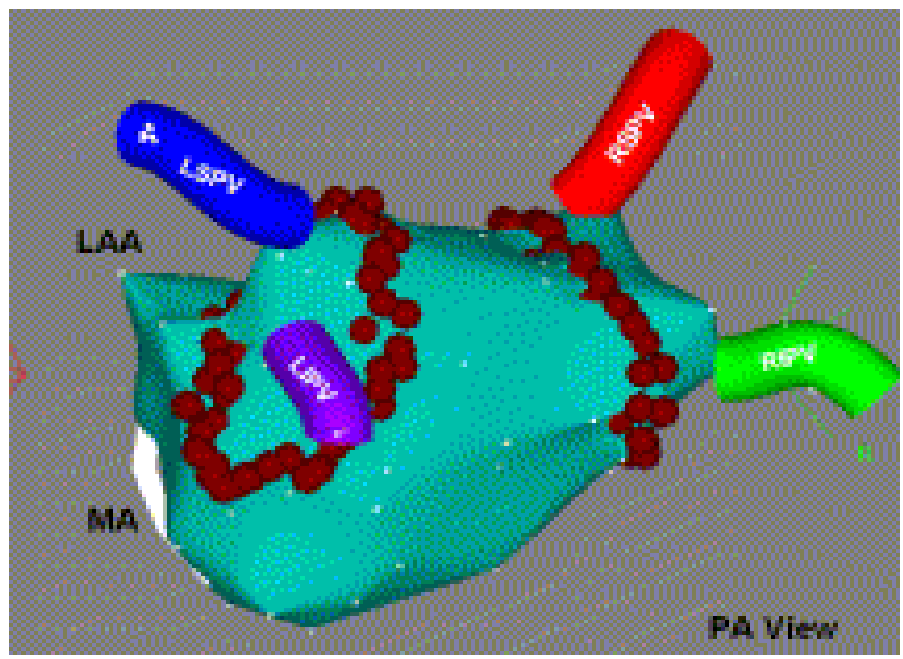

Abbildung 07: Zirkuläre PVA der jeweils rechten bzw. linken PV unter Verwendung des elektroanatomischen Mappings (Carto). LAA = left atrial appendage; $\mathrm{LI}=$ left inferior; MA $=$ mitral annulus; $\mathrm{PA}=$ pulmonary artery; $\mathrm{RI}=$ right inferior (Cheema et al. 2007). 


\subsection{Statistische Methoden}

Die statistischen Berechnungen wurden mit der Statistiksoftware SPSS Statistics für Windows (Version 16.0, SPSS Inc., Chicago Illinois, USA) angefertigt. Zur Analyse der diagnostischen Genauigkeit der MDCT, verglichen mit der invasiven Koronarangiographie, wurden die Sensitivität, Spezifität sowie der positiv und negativ prädiktive Wert (PPW, NPW) ermittelt. Für alle statistischen Berechnungen wurde ein pWert von $<0,05$ als signifikant gewertet.

\subsubsection{Chi-Quadrat-Unabhängigkeitstest}

Mittels Chi-Quadrat-Test (X2) kann die Unabhängigkeit zweier kategorialer Merkmale getestet werden. Es erfolgt der Vergleich der erwarteten theoretischen Häufigkeiten - die aufgestellte und zu prüfende Hypothese wird als Nullhypothese mit dem Symbol Ho bezeichnet (Kobelt und Steinhausen 2006) - mit den beobachteten, empirischen Häufigkeiten. Hierdurch wird eine Prüfgröße ( $\left.x^{2}\right)$ errechnet, die asymptotisch eine ChiQuadrat-Verteilung hat. Abhängig von dem gewählten Signifikanzniveau kann nun die Nullhypothese verworfen oder beibehalten werden.

\subsubsection{Sensitivität, Spezifität und Vorhersagewert}

Zur Beurteilung der Qualität eines neuen diagnostischen Verfahrens betrachtet man, getrennt für gesunde und erkrankte Personen, die Wahrscheinlichkeit für eine richtige Vorhersage mittels der neuen Maßnahme. Zum Vergleich zweier diagnostischer Maßnahmen können die Werte für Sensitivität und Spezifität herangezogen werden.

Die diagnostische Sensitivität gibt die Anzahl wahrer positiver Ergebnisse bei Patienten an, bei denen die Krankheit mit Sicherheit besteht. Damit ist sie ein Wahrscheinlichkeitsmaß, Kranke richtig zu erfassen (Altman 1991). Bei dieser Studie bedeutet dies, wie sicher eine Stenose > $50 \%$ in der MDCT erkannt wird, die auch in der invasiven Koronarangiographie als hämodynamisch relevant angesehen wird.

Die diagnostische Spezifität gibt die Anzahl wahrer negativer Ergebnisse bei Patienten an, bei denen die Krankheit nicht besteht (Altman 1991). Damit ist sie also ein Wahrscheinlichkeitsmaß, Gesunde richtig auszuschließen. Auf unsere Studie übertragen 
bedeutet dies, wie sicher ein Patient ohne Stenose $>50 \%$ als gesund erkannt wird. Der positive Vorhersagewert (= prädiktive Wert des positiven Befundes) bezeichnet die Wahrscheinlichkeit, dass ein positives Test-Ergebnis effektiv auf einem positiven Ergebnis beruht, also wie sicher eine Stenose > $50 \%$ in der MDCT auch in der invasiven Koronarangiographie bestätigt wird (Altman 1991). Umgekehrt bezeichnet der negative Vorhersagewert (= prädiktive Wert des negativen Befundes) die Wahrscheinlichkeit, dass ein negatives Ergebnis auch tatsächlich negativ ist, also wie sicher eine nicht vorliegende Stenose $>50 \%$ in der MDCT auch als solche in der invasiven Koronarangiographie bestätigt wird.

Eine optimale diagnostische Maßnahme weist Werte von jeweils $100 \%$ für Spezifität und Sensitivität auf, somit wären alle Vorhersagen richtig. Ein Verfahren ist eindeutig zu präferieren, falls es sich sowohl durch eine höhere Sensitivität als auch durch eine höhere Spezifität auszeichnet. Die erläuterten Begriffe sollen zur Veranschaulichung in einer Vierfeldertafel dargestellt werden. Der Begriff Stenose soll im Folgenden eine Engstelle > $50 \%$ bezeichnen.

Tabelle 06: Spezifität, Sensitivität und Vorhersagewerte (Altman 1991).

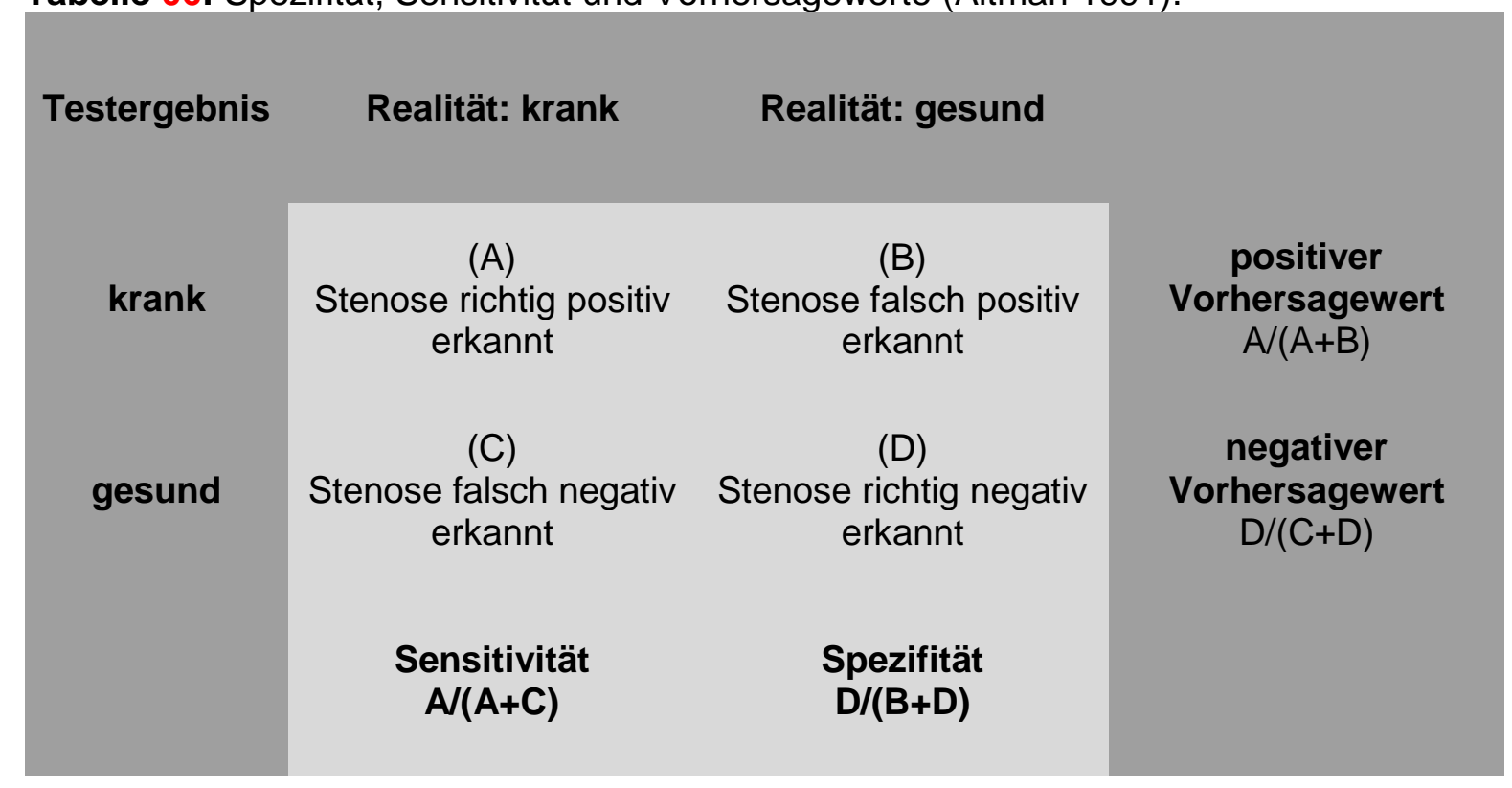




\section{Ergebnisse}

In diese Studie wurden 181 Patienten aus der Abteilung Kardiologie und Pneumologie der Universitätsklinik Göttingen eingeschlossen, bei denen vor einer PVA eine MDCT sowie bei gegebener Indikation eine invasive Koronarangiographie durchgeführt wurden.

\subsection{Risikofaktoren für eine KHK}

Zur individuellen Risikostratifizierung des Gesamtrisikos für kardiovaskuläre Ereignisse wurde in unserer Studie initial mittels Duke Clinical Score die Wahrscheinlichkeit für eine Stenose in den Koronararterien evaluiert (Kumamaru et. al 2014). Dieses Punktesystem beinhaltet Art der thorakalen Beschwerden, das Alter, das Geschlecht sowie die traditionellen Risikofaktoren (Nikotinkonsum in den letzten 5 Jahren, Gesamtcholesterin, Diabetes mellitus, vorangegangener Herzinfarkt, Q-Zacken oder persistierende STStrecken Veränderungen im EKG) (Pryor et al. 1993; Gibbons et al. 2002). Die folgende Tabelle 07 zeigt, welche Risikofaktoren bei unserem Patientenkollektiv vorlagen.

Tabelle 07: Kardiovaskuläre Risikofaktoren der Studienpopulation.

\begin{tabular}{cc} 
Risikofaktoren & $\mathbf{n = 1 8 1}$ \\
\hline Arterieller Hypertonus (n/\%) & $102(56)$ \\
Diabetes mellitus ( $/ \%)$ & $9(5)$ \\
Adipositas ( $/ \%)$ & $57(31)$ \\
Nikotinkonsum (n/\%) & $16(9)$ \\
Hyperlipidämie (n/\%) & $35(19)$
\end{tabular}




\subsection{Bildqualität}

Alle CT-Datensätze der 181 Patienten (100 \%) hatten diagnostische Qualität und konnten zur Gefäßanalyse verwendet werden. Somit wurden insgesamt 1810 Segmente ausgewertet. Entsprechend unseren o.g. Qualitätskriterien wurden 75 \% der Aufnahmen als gut, $23 \%$ als moderat und $2 \%$ als ausreichend beurteilbar klassifiziert.

\subsection{Selektive Gefäßanalyse}

\subsubsection{Ergebnisse aller Segmente in der MDCT-Koronarangiographie}

Insgesamt wurden mittels MDCT 1810 Koronarsegmente mit einem Durchmesser $\geq 1,5 \mathrm{~mm}$ evaluiert. Hierbei wurden in 35 der 1810 Segmente (2 \%) hämodynamisch relevante Stenosen detektiert. Diese 35 Stenosen verteilten sich auf 17 der 181 Patienten (9\%). Basierend auf der MDCT-Untersuchung wurde bei 13 der 181 Patienten (7\%) eine 1-Gefäß-Erkrankung, bei 3 der 181 Patienten (2\%) eine 2-Gefäß-Erkrankung und bei 1 von 181 Patienten (1\%) eine 3-Gefäß-Erkrankung detektiert. Die genaue Verteilung der gesamten in der MDCT detektierten Stenosen ist in den Tabellen 08 bis 10 dargestellt. 
Tabelle 08: Anzahl detektierter Stenosen $>50 \%$ in den Koronararterien aufgeteilt nach einzelnen Segmenten.

\begin{tabular}{|c|c|c|}
\hline Segment & Anzahl Segmente & $\begin{array}{c}\text { Stenosen MDCT- } \\
\text { Koronarangiographie }\end{array}$ \\
\hline Segmente insgesamt & 1810 & 35 \\
\hline Arteria coronaria sinistra & 181 & 1 \\
\hline \multicolumn{3}{|l|}{$\begin{array}{l}\text { Ramus interventricularis } \\
\text { anterior }\end{array}$} \\
\hline Proximal & 181 & 11 \\
\hline Medial & 181 & 8 \\
\hline Distal & 181 & 1 \\
\hline \multicolumn{3}{|l|}{ Ramus circumflexus } \\
\hline Proximal & 181 & 5 \\
\hline 1. Marginaler Ast & 181 & 2 \\
\hline Distal & 181 & 0 \\
\hline \multicolumn{3}{|l|}{ Arteria coronaria dextra } \\
\hline Proximal & 181 & 2 \\
\hline Medial & 181 & 4 \\
\hline Distal & 181 & 1 \\
\hline
\end{tabular}

Tabelle 09: Anzahl detektierter Stenosen > 50\% in den Koronararterien aufgeteilt nach Hauptgefäßen.

\begin{tabular}{cc|} 
Koronararterie & Stenosen MDCT-Koronarangiographie \\
\hline Arteria coronaria sinistra & 1 \\
Ramus interventricularis anterior & 20 \\
Ramus circumflexus & 7 \\
Arteria coronaria dextra & 7
\end{tabular}


Tabelle 10: Detektion einer KHK in der MDCT-Koronarangiographie.

KHK in MDCT insgesamt

Ein-Gefäß-KHK

Zwei-Gefäß-KHK

Drei-Gefäß-KHK
Anzahl

13

3

1

\subsubsection{Detektion von Stenosen in der invasiven Koronarangiographie}

Mit Hilfe der invasiven Koronarangiographie konnten die Befunde aus der MDCTUntersuchung hinsichtlich der Detektion von hämodynamisch relevanten und (noch) nicht hämodynamisch relevanten Stenosen von 86 Patienten (48 \%) evaluiert werden. Insgesamt konnten somit 860 Segmente (48\%) direkt miteinander verglichen werden. Aufgrund von Stentartefakten wurden 6 Segmente aus dem direkten Vergleich ausgeschlossen, so dass nur 854 (47\%) Segmente in den direkten Vergleich eingingen. Laut invasiver Koronarangiographie wiesen 1 \% (10 von 854) der evaluierten Segmente hämodynamisch relevante Stenosen auf, diese verteilten sich auf $9 \%$ der Patienten (8 von 86). Von diesen 8 Patienten hatten laut invasiver Koronarangiographie $75 \%$ eine EinGefäß-KHK und 25 \% eine Zwei-Gefäß-KHK. Neben der Dokumentation von hämodynamisch relevanten Stenosen wurden ebenfalls hämodynamisch nicht relevante Stenosen erfasst (<50\% Lumeneinengung). Diese verteilten sich auf $17 \%$ der Patienten (15 von 86).

\subsection{Vergleich von MDCT-Koronarangiographie und invasiver Koronarangiographie}

Die invasive Koronarangiographie diente bei unserem Patientenkollektiv als Goldstandard und Vergleichsmethode zur MDCT. Somit konnten anhand der invasiven Koronarangiographie im direkten Vergleich die Sensitivität und die Spezifität sowie der NPW und der PPW der MDCT evaluiert werden. In der invasiven Koronarangiographie wurden bei 854 Segmenten insgesamt 10 Läsionen mit einer Lumeneinengung über 50 \% detektiert. 
Im Vergleich dazu waren es in der MDCT-Koronarangiographie 24 Stenosen. Tabelle 11 zeigt die insgesamt ermittelten relevanten Stenosen der jeweiligen Verfahren.

Tabelle 11: Anzahl detektierter Stenosen > $50 \%$ in MDCT und invasiver Koronarangiographie (bezogen auf die einzelnen Segmente der Koronararterien).

\begin{tabular}{|c|c|c|c|}
\hline Segment & $\begin{array}{c}\text { Anzahl } \\
\text { Segmente }\end{array}$ & $\begin{array}{c}\text { Anzahl Stenosen } \\
\text { invasive } \\
\text { Koronarangiographie }\end{array}$ & $\begin{array}{c}\text { Anzahl Stenosen } \\
\text { MDCT- } \\
\text { Koronarangiographie }\end{array}$ \\
\hline Segmente insgesamt & 854 & 10 & 24 \\
\hline $\begin{array}{l}\text { Arteria coronaria } \\
\text { sinistra }\end{array}$ & 86 & 0 & 1 \\
\hline \multicolumn{4}{|l|}{$\begin{array}{c}\text { Ramus } \\
\text { interventricularis } \\
\text { anterior }\end{array}$} \\
\hline Proximal & 86 & 3 & 7 \\
\hline Medial & 86 & 1 & 5 \\
\hline Distal & 86 & 1 & 1 \\
\hline \multicolumn{4}{|l|}{ Ramus circumflexus } \\
\hline Proximal & 85 & 2 & 3 \\
\hline 1. Marginaler Ast & 85 & 0 & 1 \\
\hline Distal & 85 & 0 & 0 \\
\hline \multicolumn{4}{|l|}{$\begin{array}{c}\text { Arteria coronaria } \\
\text { dextra }\end{array}$} \\
\hline Proximal & 84 & 0 & 1 \\
\hline Medial & 85 & 2 & 4 \\
\hline Distal & 86 & 1 & 1 \\
\hline
\end{tabular}


Tabelle 12 zeigt zusammenfassend die Anzahl der signifikanten Stenosen in den einzelnen Koronararterien.

Tabelle 12: Anzahl detektierter Stenosen > $50 \%$ in MDCT und invasiver Koronarangiographie (bezogen auf die Koronararterien).

\begin{tabular}{ccc} 
Gefäß & $\begin{array}{c}\text { Anzahl Stenosen } \\
\text { invasive } \\
\text { Koronarangiographie }\end{array}$ & $\begin{array}{c}\text { Anzahl Stenosen } \\
\text { MDCT- } \\
\text { Koronarangiographie }\end{array}$ \\
\hline $\begin{array}{c}\text { Arteria coronaria sinistra } \\
\text { Ramus interventricularis } \\
\text { anterior }\end{array}$ & 0 & 1 \\
Ramus circumflexus & 5 & 13 \\
Arteria coronaria dextra & 2 & 4 \\
& 3 & 6
\end{tabular}

\subsubsection{Unterschiede in der Detektion von Stenosen}

Es ist hervorzuheben, dass alle signifikanten Stenosen in den hämodynamisch relevanten Gefäßabschnitten richtig positiv von der MDCT detektiert wurden. Dagegen wurde in dem proximalen Anteil des RCX eine hämodynamisch relevante Stenose (40\% Stenosierung in der MDCT und $70 \%$ Stenosierung in der invasiven Koronarangiographie) übersehen und eine weitere richtig positiv detektiert. Abb. 08 zeigt, nach welchem Schema die Klassifikation der Ergebnisse erfolgte.
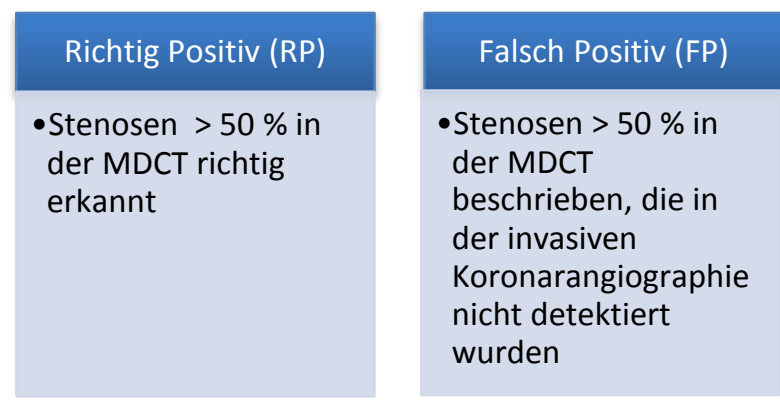

\section{Richtig Negativ (RN)}

-weder in der MDCT noch in der invasiven Koronarangiographie wurde eine Stenose > $50 \%$ detektiert
Falsch Negativ (FN)

-in der MDCT wurde keine Stenose $>50 \%$ detektiert, obwohl in der invasiven Koronarangiographie eine Stenose $>50 \%$ vorlag

Abbildung 08: Klassifikation der Ergebnisse. 
Tabellen 13 und 14 zeigen die Anzahl der richtig bzw. falsch erkannten Stenosen in der MDCT, bezogen auf die einzelnen Segmente der Koronararterien.

Tabelle 13: Anzahl richtig bzw. falsch detektierter Stenosen > $50 \%$ in MDCT (bezogen auf die einzelnen Segmente der Koronararterien).

\begin{tabular}{|c|c|c|c|c|}
\hline Segment & $\begin{array}{l}\text { Richtig } \\
\text { Positiv }\end{array}$ & $\begin{array}{l}\text { Falsch } \\
\text { Positiv }\end{array}$ & $\begin{array}{l}\text { Richtig } \\
\text { Negativ }\end{array}$ & $\begin{array}{l}\text { Falsch } \\
\text { Negativ }\end{array}$ \\
\hline Segmente insgesamt & 9 & 15 & 829 & 1 \\
\hline $\begin{array}{l}\text { Arteria coronaria } \\
\text { sinistra }\end{array}$ & 0 & 1 & 85 & 0 \\
\hline \multicolumn{5}{|l|}{$\begin{array}{l}\text { Ramus } \\
\text { interventricularis } \\
\text { anterior }\end{array}$} \\
\hline Proximal & 3 & 4 & 79 & 0 \\
\hline Medial & 1 & 4 & 81 & 0 \\
\hline Distal & 1 & 0 & 85 & 0 \\
\hline \multicolumn{5}{|l|}{ Ramus circumflexus } \\
\hline Proximal & 1 & 2 & 81 & 1 \\
\hline 1. Marginaler Ast & 0 & 1 & 84 & 0 \\
\hline Distal & 0 & 0 & 85 & 0 \\
\hline \multicolumn{5}{|l|}{ Arteria coronaria dextra } \\
\hline Proximal & 0 & 1 & 83 & 0 \\
\hline Medial & 2 & 2 & 81 & 0 \\
\hline Distal & 1 & 0 & 85 & 0 \\
\hline
\end{tabular}


Tabelle 14: Anzahl richtig bzw. falsch detektierter Stenosen $>50 \%$ in der MDCT (bezogen auf die Koronararterien).

\begin{tabular}{c|cccc}
\hline Gefäß & $\begin{array}{c}\text { Richtig } \\
\text { Positiv }\end{array}$ & $\begin{array}{c}\text { Falsch } \\
\text { Positiv }\end{array}$ & $\begin{array}{c}\text { Richtig } \\
\text { Negativ }\end{array}$ & $\begin{array}{c}\text { Falsch } \\
\text { Negativ }\end{array}$ \\
\hline $\begin{array}{c}\text { Arteria coronaria } \\
\text { sinistra } \\
\begin{array}{c}\text { Ramus } \\
\text { interventricularis } \\
\text { anterior }\end{array}\end{array}$ & 0 & 1 & 85 & 0 \\
$\begin{array}{c}\text { Ramus circumflexus } \\
\begin{array}{c}\text { Arteria coronaria } \\
\text { dextra }\end{array}\end{array}$ & 1 & 8 & 245 & 0 \\
\hline
\end{tabular}

Die eine in der MDCT nicht korrekt klassifizierte Stenose im Bereich des proximalen RCX ist möglicherweise damit zu erklären, dass in der MDCT-Analyse die Stenose mit $<50 \%$ quantifiziert wurde (40\%) und folglich als nicht signifikant eingestuft wurde. Somit wurde diese Stenose nicht übersehen, sondern lediglich anhand der Darstellung in der MDCT anders beurteilt. Zusätzlich ist zu erwähnen, dass dieser Patient aufgrund einer anderen Stenose in dem RIVA bereits richtig positiv als Patient mit einer KHK klassifiziert wurde. Der umgekehrte Fall lag bei den in der MDCT falsch positiv bewerteten Ergebnissen (15 Segmente) vor. Diesbezüglich wurden in der invasiven Koronarangiographie meist ebenfalls Stenosen detektiert, diese jedoch mit $<50 \%$ Lumeneinengung als nicht signifikant eingestuft.

\subsubsection{Sensitivität und Spezifität}

Tabellen 15 und 16 zeigen die Sensitivität und die Spezifität der MDCTKoronarangiographie bezogen auf die einzelnen Segmente der Koronararterien. 
Tabelle 15: Sensitivität und Spezifität bezogen auf die einzelnen Segmente der Koronararterien (bei "," wurden aufgrund von nicht vorhandenen Stenosen keine statistischen Auswertungen vorgenommen).

\begin{tabular}{ccc} 
Segment & Sensitivität (\%) & Spezifität (\%) \\
\hline Segmente insgesamt & 90 & 98 \\
$\begin{array}{c}\text { Arteria coronaria sinistra } \\
\text { Ramus interventricularis } \\
\text { anterior } \\
\text { Proximal }\end{array}$ & 100 & 99 \\
Medial & 100 & 95 \\
Distal & 100 & 95 \\
\hline Ramus circumflexus & 100 & 100 \\
Proximal & & 98 \\
1. Marginaler Ast & 50 & 100 \\
Distal & - & 100 \\
Distal & 100 & 99 \\
\hline Prteria coronaria dextra & - & 98 \\
Medial & 100 & 99 \\
\hline
\end{tabular}

Tabelle 16: Sensitivität und Spezifität bezogen auf die Koronararterien.

\begin{tabular}{ccc} 
Gefäß & Sensitivität (\%) & Spezifität (\%) \\
\hline $\begin{array}{ccc}\text { Arteria coronaria sinistra } \\
\text { Ramus interventricularis } \\
\text { anterior }\end{array}$ & 100 & 99 \\
Ramus circumflexus & 100 & 97 \\
Arteria coronaria dextra & 50 & 99 \\
& 100 & 99
\end{tabular}




\subsubsection{Positiver und negativer Vorhersagewert}

Zur Einschätzung der Aussagekraft der MDCT-Untersuchungen zeigt Tabelle 17, bezogen auf die einzelnen Segmente der Koronararterien die positiven und negativen Vorhersagewerte.

Tabelle 17: Positiver und negativer Vorhersagewert der MDCT-Koronarangiographie bezogen auf die einzelnen Segmente.

\begin{tabular}{|c|c|c|}
\hline Segment & $\begin{array}{c}\text { Positiver Vorhersagewert } \\
(\%)\end{array}$ & $\begin{array}{c}\text { Negativer Vorhersagewert } \\
(\%)\end{array}$ \\
\hline Segmente insgesamt & 38 & 100 \\
\hline Arteria coronaria sinistra & 0 & 100 \\
\hline \multicolumn{3}{|l|}{$\begin{array}{c}\text { Ramus interventricularis } \\
\text { anterior }\end{array}$} \\
\hline Proximal & 43 & 100 \\
\hline Medial & 20 & 100 \\
\hline Distal & 100 & 100 \\
\hline \multicolumn{3}{|l|}{ Ramus circumflexus } \\
\hline Proximal & 33 & 99 \\
\hline 1. Marginaler Ast & 0 & 100 \\
\hline Distal & 0 & 100 \\
\hline \multicolumn{3}{|l|}{ Arteria coronaria dextra } \\
\hline Proximal & 0 & 100 \\
\hline Medial & 50 & 100 \\
\hline Distal & 100 & 100 \\
\hline
\end{tabular}


Tabelle 18 zeigt den positiv und negativ prädiktiven Wert der MDCT-Koronarangiographie bezogen auf die einzelnen Koronararterien.

Tabelle 18: Positiv und negativ prädiktiver Wert der MDCT-Koronarangiographie bezogen auf die einzelnen Koronararterien.

\begin{tabular}{ccc} 
Gefäß & $\begin{array}{c}\text { Positiver Vorhersagewert } \\
(\%)\end{array}$ & $\begin{array}{c}\text { Negativer Vorhersagewert } \\
(\%)\end{array}$ \\
\hline $\begin{array}{c}\text { Arteria coronaria sinistra } \\
\text { Ramus interventricularis } \\
\quad \text { anterior }\end{array}$ & 0 & 100 \\
Ramus circumflexus & 54 & 100 \\
Arteria coronaria dextra & 11 & 100 \\
& 50 & 100
\end{tabular}

Mittels MDCT-Koronarangiographie konnten 829 von 844 (98\%) Koronarsegmenten, die laut invasiver Koronarangiographie keine Stenosen aufwiesen, auch als solche korrekt detektiert werden. Jeder Patient mit einer 1-Gefäß-KHK ( $n=6)$ wurde in der MDCT richtig beurteilt. Bei zwei weiteren Patienten mit jeweils einer 2-Gefäß-KHK wurden diese in der MDCT-Koronarangiographie als 1- bzw. 3-Gefäß-KHK eingestuft. Damit machen die 1Gefäß-Erkrankungen 75 \% (6 von 8) und die 2-Gefäß-Erkrankungen 25 \% (2 von 8) der KHK-Klassifikationen in der invasiven Koronarangiographie aus. Insgesamt wurden mit der MDCT-Koronarangiographie alle Patienten mit einer signifikanten Stenose in einem oder mehreren Segmenten auch als solche richtig positiv erkannt. Tabelle 19 zeigt den Schweregrad der KHK basierend auf der Anzahl der betroffenen Gefäße.

Tabelle 19: Anzahl der verschiedenen KHK-Klassifikationen in MDCT und invasiver Koronarangiographie.

\begin{tabular}{ccc} 
KHK-Klassifikation & $\begin{array}{c}\text { MDCT- } \\
\text { Koronarangiographie }\end{array}$ & $\begin{array}{c}\text { Invasive } \\
\text { Koronarangiographie }\end{array}$ \\
\hline Ein-Gefäß-KHK & 7 & 6 \\
Zwei-Gefäß-KHK & 0 & 2 \\
Drei-Gefäß-KHK & 1 & 0
\end{tabular}


Alle Patienten mit einer 1-Gefäß-KHK erhielten eine perkutane Koronarintervention (PKI). Bei einem der oben erwähnten Patienten mit einer 2-Gefäß-KHK war eine Stentimplantation in beiden befallenen Gefäßen notwendig, während der zweite Patienten mit der 2-Gefäß-KHK lediglich eine Stentimplantation im RIVA benötigte.

\subsection{Abbildungen}

Abb. 09 zeigt ein Beispiel für eine richtig positiv erkannte Stenose mittels MDCTKoronarangiographie: Ein 58-jähriger Patient mit paroxysmalem VHF und Dyspnoe unterzog sich einer koronaren Routine-MDCT-Angiographie vor PVA sowie einer konventionellen Koronarangiographie. Es zeigt sich eine interventionsbedürftige 70 \%ige Stenose des RIVA. Zu sehen sind verschiedene Rekonstruktionen, als zweidimensionale Darstellung wird die MPR und als 3D-Darstellung die VRT gewählt. 


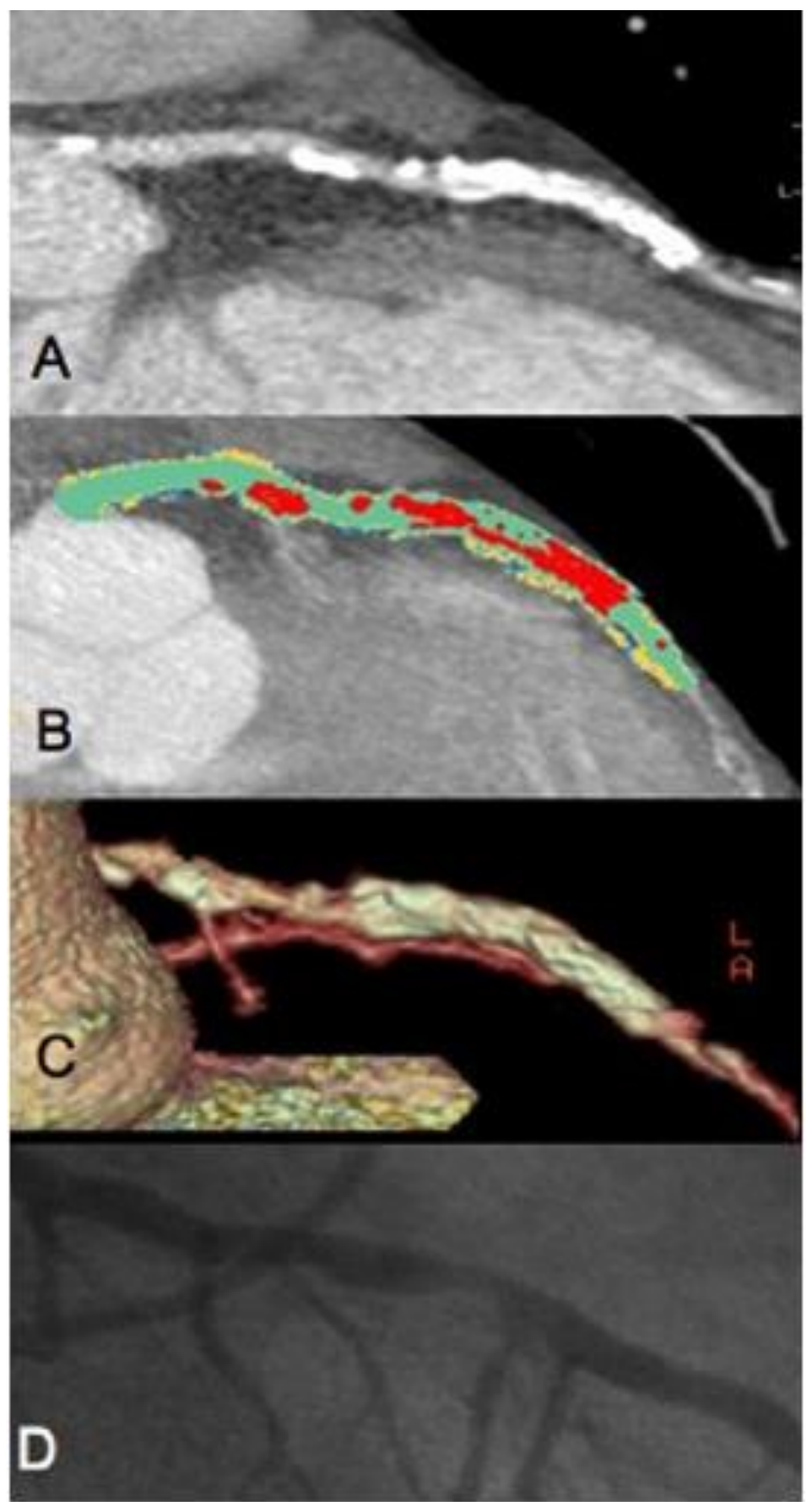

Abbildung 09: 58 Jahre alter Patient mit paroxysmalem Vorhofflimmern, Dyspnoe und 70 \%iger Stenose des RIVA. (A) Multiplanare Rekonstruktion in der MDCT sowie die korrespondierende farblich unterlegte Gefäßanalyse mit den rot markierten signifikanten Stenosen des proximalen RIVA (B). (C) Überblick über die Anatomie des RIVA mittels VRT. (D) Invasive Koronarangiographie. 
Abb. 10 zeigt ein typisches Beispiel für eine richtig positiv erkannte Zwei-Gefäß-KHK mittels MDCT-Angiographie: Ein 56-jähriger Patient mit permanentem VHF, arterieller Hypertonie, Adipositas I $^{\circ}\left(\mathrm{BMI} 32 \mathrm{~kg} / \mathrm{m}^{2}\right)$ sowie Hyperlipidämie. Zu sehen ist die $90 \%$ ige Stenose in dem RIVA, weiterhin lag eine 50 \%ige Stenose in der proximalen ACD vor.

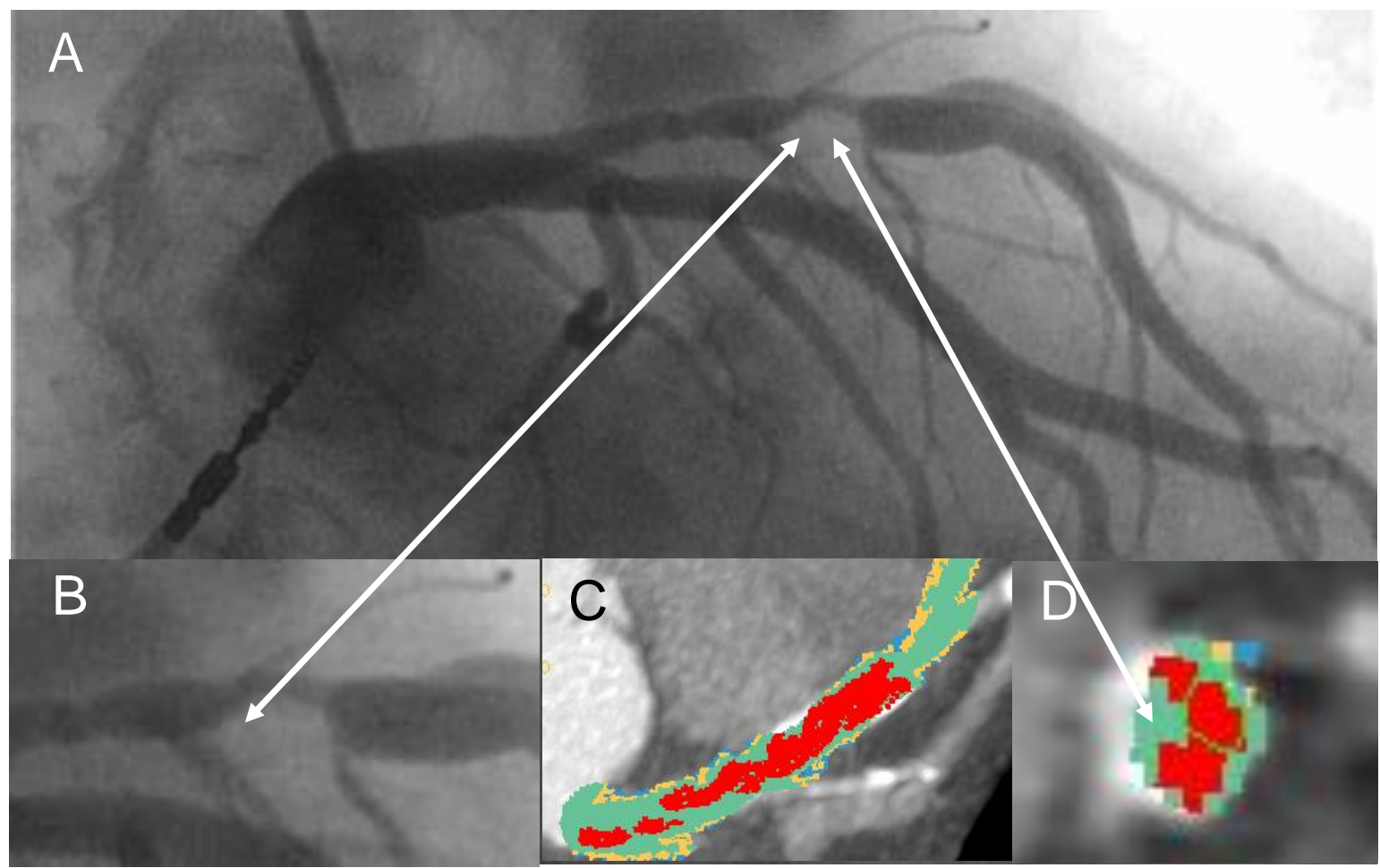

Abbildung 10: (A) Invasive Koronarangiographie mit (B) Vergrößerung der signifikanten interventionsbedürftigen Stenose in dem proximalen RIVA $(90 \%)$. Selbige Stenose rot markiert in der MDCT Branch View (C) sowie in der X-Section Darstellung (D). 
Abb. 11 zeigt eine mittels MDCT detektierte signifikante RIVA-Stenose bei einem 64jährigen Patienten mit einer 1-Gefäß-KHK und paroxysmalem VHF. Als Risikofaktoren lagen bei dem Patienten ein Nikotinkonsum sowie eine Hyperlipidämie vor.

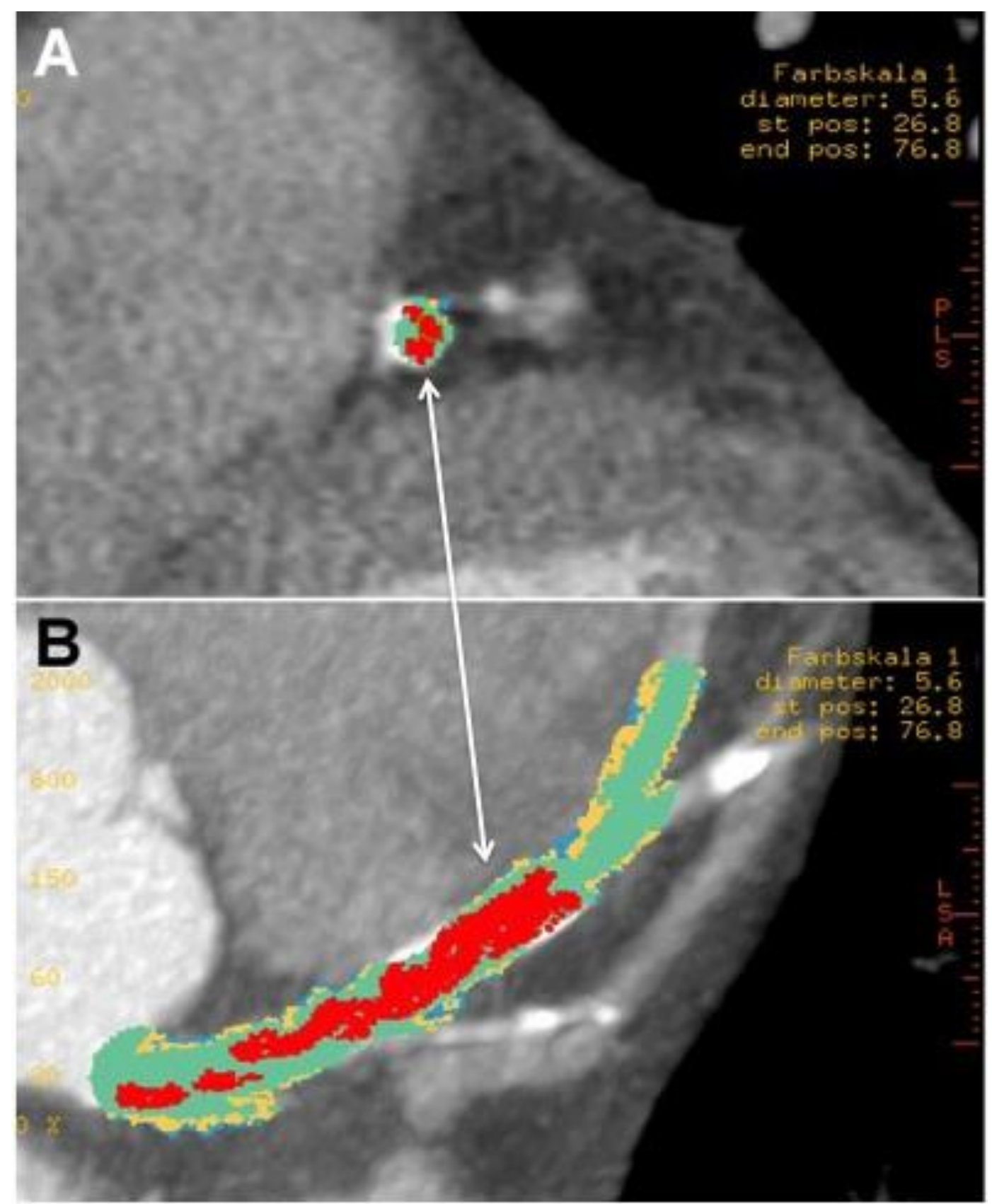

Abbildung 11: Patienten mit 1-Gefäß-KHK und signifikanter Stenose im RIVA. Darstellung in Branch View (A) und in X-Section (B). Rot markiert zu sehen ist die Lumeneinengung. 
Abb. 12 zeigt eine signifikante Stenose der proximalen ACD eines Patienten mit niedriger Prätest-Wahrscheinlichkeit für das Vorliegen einer KHK. Als Risikofaktoren bestanden ein persistierendes VHF sowie eine arterielle Hypertonie.

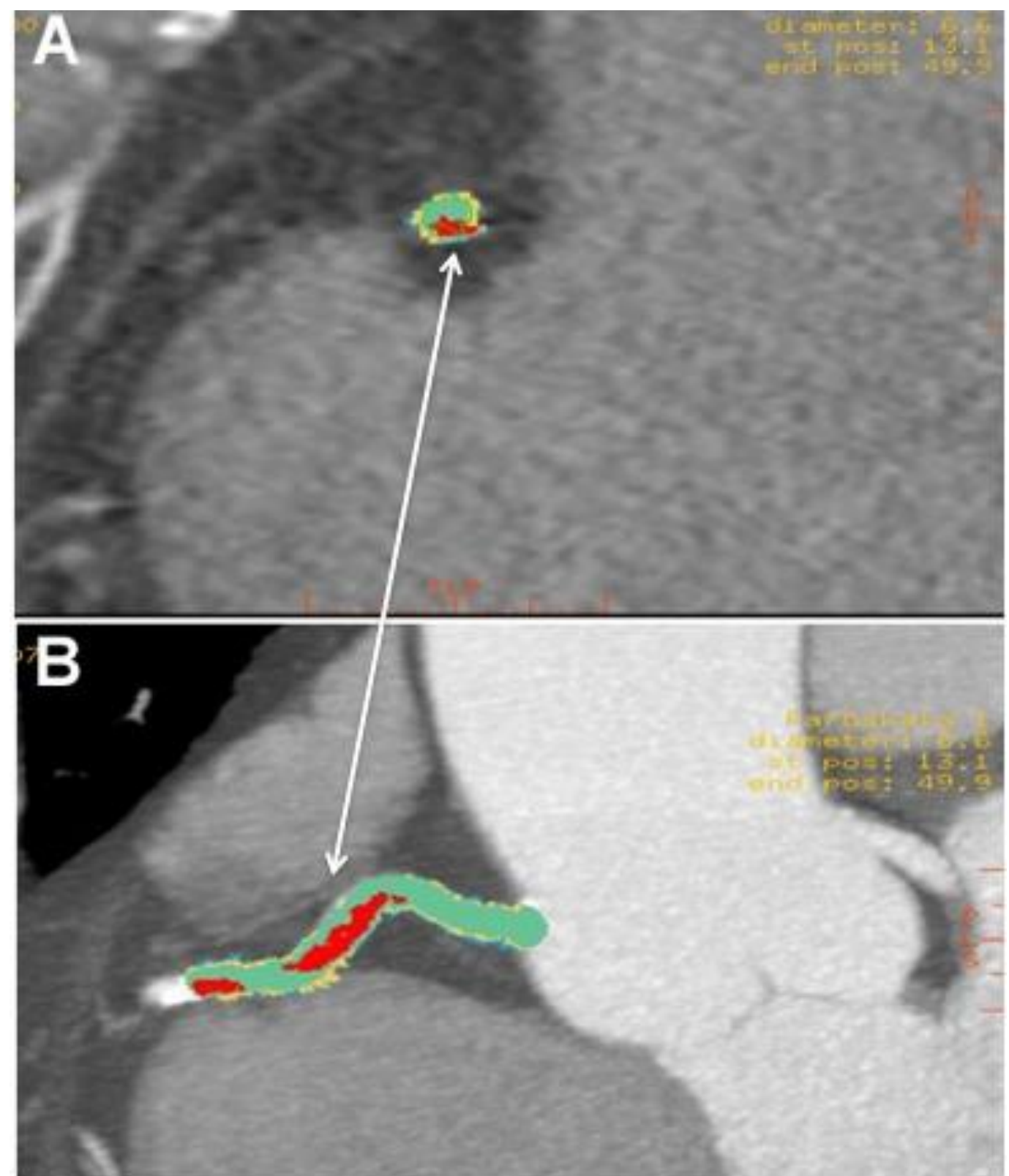

Abbildung 12: Stenose der ACD eines Patienten mit einer 1-Gefäß-KHK in Branch View (A) und X-Section Darstellung (B). Rot markiert zu sehen ist die Lumeneinengung. 


\section{Diskussion}

\subsection{Hintergrund dieser Studie}

Aufgrund der technischen Weiterentwicklungen der letzten Jahre nimmt die MDCT eine zunehmend wichtigere Rolle auf dem Gebiet der nichtinvasiven Diagnostik des Herzens ein. Insbesondere ist hierbei die Integration anatomischer Bilddaten in 3D-Navigationssysteme mittlerweile fester Bestandteil elektrophysiologischer Untersuchungen.

Diesbezüglich ist die vorliegende Arbeit die erste, die den Stellenwert der MDCTKoronarangiographie bei Patienten mit symptomatischem VHF und Indikation zur Katheterablation im direkten Vergleich mit der invasiven Koronarangiographie evaluiert.

Die Kernaussage unserer Studie ist, dass sich mittels der routinemäßig vor PVA generierten Bilddaten der 64-Zeilen-MDCT neben der für die Ablation wichtigen LAAnatomie die klinisch bedeutsamen Abschnitte des Koronarbaumes visualisieren lassen. Zum einen zeigen unsere Daten, dass die MDCT mit einem NPW von $100 \%$ eine adäquate Methode zum sicheren Ausschluss von hämodynamisch relevanten Stenosen (> $50 \%$ ) ist. Zum anderen konnten in unserer Studie mittels MDCT alle KHK-Patienten mit signifikanten Stenosen in einem oder mehreren Segmenten auch als solche erkannt werden. Damit verdeutlichen unsere Ergebnisse, dass die 64-Zeilen-MDCT, im Vergleich zu früheren kardialen Bildgebungen nochmals verbessert und entgegen älteren Studien (Becker et al. 2000) auch in einem nicht streng selektierten Patientengut zuverlässig einsetzbar ist. Entsprechend findet die CTA in den 2013 überarbeiteten ESC-Leitlinien zum Management des akuten Koronarsyndroms nun erstmals Berücksichtigung und wird als eine Möglichkeit zum Ausschluss einer relevanten KHK bei Patienten mit niedrigem Risiko (u.a. keine EKG-Veränderungen, keine Troponinerhöhung etc.) erwähnt (Montalescot et al. 2013). 


\subsection{Vergleich mit anderen Untersuchungen}

\subsubsection{Studienlage zum Thema}

Beleuchtet man die Historie der kardiovaskulären Diagnostik, zeigt sich, dass die kardiale CT in einem Zeitfenster von weniger als zehn Jahren einen deutlichen Innovationsschub vollzogen hat. Seit Einführung der 4-/16- und vor allem der 64-Zeilen-CT konnte eine große Anzahl von mono- und multizentrischen Studien die hohe diagnostische Genauigkeit der CTA belegen, wobei hierbei die besondere Stärke im Ausschluss von relevanten Koronarstenosen liegt. VHF galt jedoch in der Vergangenheit in vielen Studien dieser Art als Ausschlusskriterium (Achenbach et al. 2000; Achenbach et al. 2001; Herzog et al. 2002; Hoffmann et al. 2004; Leber et al. 2003; Leber et al. 2004; Niemann et al. 2002a; Ropers et al. 2003; Schoepf et al. 2004). Dies ist damit zu erklären, dass eine hohe HF, im Besonderen Arrhythmien, Bildartefakte und damit deutlich schlechtere Bildqualitäten bewirken. Die Güte der bildlichen Darstellung des Herzens hat jedoch, dank stetiger Weiterentwicklung und Neuerungen auf diesem Gebiet, deutlich zugenommen. So erlaubt diese nun nicht nur die Darstellung der Koronarien bei höheren HF, sondern auch bei Arrhythmien (Ropers et al. 2003).

\subsubsection{Vergleich der Ergebnisse mit anderen Studien}

Betrachtet man die ersten Studien, in denen die Ergebnisse der 4-Zeilen-MDCT bezüglich der Detektion signifikanter Koronararterienstenosen mit denen der invasiven Koronarangiographie verglichen wurden (Tabelle 20), so ergaben sich eine Sensitivität von 81-91 \% sowie eine Spezifität von 76-96 \%. (Niemann et al. 2002a; Achenbach et al. 2001; Giesler et al. 2002; Kopp et al. 2002). Diese Ergebnisse sind für Kollektive mit Sinusrhythmus ermittelt worden (mittlere HF $<65 \mathrm{~S} / \mathrm{min}$ ), wobei auch hier aufgrund einer mangelhaften Bildqualität nur ein geringer Teil der Koronararterien beurteilbar war. Beschränkt wurde sich in der Beurteilung auf die proximalen und medialen Segmente (meistens die Segmente 1-3, 5-8 und 11), so dass beispielsweise in der Studie von Achenbach et al. lediglich $68 \%$ der untersuchten Segmente tatsächlich analysiert werden konnten (Achenbach et al. 2001). Diesbezüglich bestätigt eine Studie von Hoffman et al. aus dem Jahre 2004, dass Sensitivität und Spezifität von der Anzahl der von der Beurteilung ausgeschlossenen Segmente abhängen: Je mehr Segmente in die 
Beurteilung mit einbezogen wurden, desto geringer waren die Werte für Sensitivität und Spezifität (Hoffmann et al. 2004). Die mittels 16-Schicht-MDCT ermittelte Sensitivität betrug $70 \%$ für die proximalen Koronarsegmente, wobei bei der Beurteilung aller Koronarsegmente eine geringere Sensitivität von $63 \%$ erzielt wurde. Die Spezifität (94\% vs. $96 \%$ ) differierte in diesem Fall deutlich weniger.

Spätere Studien mit der gegenwärtigen Generation der 64-Zeilen-MDCT erzielten aufgrund ihrer im Vergleich zu älteren Exemplaren höheren räumlichen und zeitlichen Auflösung deutlich bessere Ergebnisse. Hier variierte die Sensitivität zwischen 84-92 \%, die Spezifität wurde mit $91 \%$ beziffert. Weiterhin ergaben sich ein PPW von $80 \%$ sowie ein NPW von 97 \% (Leber et al. 2005; Raff et al. 2005). Diese Ergebnisse stehen im Einklang mit den Daten anderer Studien. Leschka et al. konnten mittels CTKoronarangiographie 165 der insgesamt 176 hämodynamisch signifikanten Stenosen in der invasiven Koronarangiographie detektieren. Die Sensitivität betrug $94 \%$, die Spezifität 97 \%, der PPW 87 \% und der NPW lag bei 99 \% (Leschka et al. 2005). Meijboom et al. untersuchten Patienten vor geplanter Herzklappen-Operation mittels CTKoronarangiographie mit dem Ziel, eine signifikante Koronarerkrankung zu detektieren. In ihrer Patientengruppe lag die Prävalenz für eine KHK bei 25,7 \%. Die Auswertung ihrer Daten ergaben eine Sensitivität von $100 \%$, eine Spezifität von $92 \%$, einen PPW von $82 \%$ und einen NPW von $100 \%$ (Meijboom et al. 2006). Diesen Ergebnissen entsprechend, gaben Stein et al. in einer Metaanalyse mehrerer älterer Studien eine mittlere Sensitivität der 64-Zeilen-CT-Koronarangiographie von $\geq 90 \%$ bezüglich der Detektion einer KHK an (Stein et al. 2008). Für den RCX (Sensitivität von $88 \%$ ), distale Segmente (Sensitivität von $80 \%$ ) und Segmente mit einem Stent $(88 \%)$ ergaben sich geringere Werte.

Hiervon abweichende Ergebnisse zeigt eine Studie von Wehrschuetz et al. aus dem Jahre 2010, die in ihrem Patientenkollektiv eine Sensitivität von 69 \% und eine Spezifität von $92 \%$ für den Nachweis von signifikanten Koronarstenosen ermittelten. Der PPW war mit $38 \%$ ebenfalls stark differierend, der NPW zeigte sich mit $98 \%$ kohärent zu vorherigen Studien. Als ursächlich für diese im Vergleich zu den anderen Daten unterschiedlichen Ergebnisse müssen zum einen der fehlende Ausschluss von Koronararterien (Arterien mit einem Durchmesser $<1,5 \mathrm{~mm}$ wurden ebenfalls evaluiert) und zum anderen der Verzicht auf bradykardisierende Medikamente wie Betablocker (Frequenzen über $65 \mathrm{~S} / \mathrm{min}$ wurden toleriert) gesehen werden. Ein weiterer Aspekt ist möglicherweise das ausgewählte Patientenkollektiv mit deutlich differierendem Risikoprofil (Wehrschuetz et al. 2010). 
Tabelle 20: Ergebnisse der 4-/64-Zeilen-Computertomographie.

\begin{tabular}{|c|c|c|c|c|c|}
\hline Studie & $\begin{array}{c}\text { Patienten- } \\
\text { zahl }\end{array}$ & $\begin{array}{c}\text { Sensitivität } \\
(\%)\end{array}$ & $\begin{array}{c}\text { Spezifität } \\
(\%)\end{array}$ & $\begin{array}{l}\text { PPV } \\
(\%)\end{array}$ & $\begin{array}{l}\text { NPV } \\
(\%)\end{array}$ \\
\hline \multicolumn{6}{|c|}{ 4-Zeilen CT } \\
\hline $\begin{array}{l}\text { Nieman et } \\
\text { al. } 2001\end{array}$ & 31 & 81 & 97 & I & I \\
\hline $\begin{array}{l}\text { Achenbach } \\
\text { et al. } 2001\end{array}$ & 64 & 85 & 76 & I & I \\
\hline $\begin{array}{c}\text { Giesler et al. } \\
2002\end{array}$ & 100 & 91 & 89 & I & I \\
\hline $\begin{array}{c}\text { Kopp et al. } \\
2002\end{array}$ & 102 & 86 & 96 & 81 & 98 \\
\hline \multicolumn{6}{|c|}{ 64-Zeilen CT } \\
\hline $\begin{array}{l}\text { Raff et al. } \\
2005\end{array}$ & 70 & 92 & 91 & 80 & 97 \\
\hline $\begin{array}{l}\text { Leber et al. } \\
\qquad 2005\end{array}$ & 59 & 84 & 91 & I & I \\
\hline $\begin{array}{l}\text { Leschka et } \\
\text { al. } 2005\end{array}$ & 67 & 94 & 97 & 87 & 99 \\
\hline $\begin{array}{c}\text { Meijboom et } \\
\text { al. } 2006\end{array}$ & 145 & 100 & 92 & 82 & 100 \\
\hline $\begin{array}{c}\text { Wehrschuetz } \\
\text { et al. } 2010\end{array}$ & 73 & 69 & 92 & 38 & 98 \\
\hline
\end{tabular}

Galten Arrhythmien zuvor als Ausschlusskriterium, untersuchte die Forschungsgruppe um Yang erstmals die Genauigkeit der Stenosedetektion mittels 64-Zeilen-MDCT bei Patienten mit VHF. Als Referenzmethode galt auch hier die invasive Koronarangiographie. Dabei lagen die Sensitivität bei 86,4 \%, die Spezifität bei 99,3\%, der PPW bei $79 \%$ sowie der NPW bei 99,6 \% (Yang et al. 2009).

Die in unserer Studie bezüglich der Detektion von Stenosen $>50 \%$ ermittelte Sensitivität von $90 \%$ und Spezifität von $98 \%$, zeigt zu früheren Studien vergleichbare Werte und beweist damit, dass auch die bis dato nur eingeschränkt untersuchte Gruppe der Patienten mit VHF von der computertomographischen Koronardiagnostik profitieren kann und trotz der Arrhythmien eine gute diagnostische Beurteilung der hämodynamisch relevanten Abschnitte der Koronararterien möglich ist (Oncel et al. 2007).

Der PPW lag in unserer Studie bei $38 \%$ und damit unter den Ergebnissen vorheriger Studien. Die Tatsache, dass somit $61 \%$ der Stenosen in der CT mittels 
Koronarangiographie nicht verifiziert werden konnten, kann damit begründet werden, dass es in der MDCT lediglich zu einer Überschätzung vorhandener Engstellen kam.

Entscheidend ist jedoch der bei unserem Patientenkollektiv mit $100 \%$ bezifferte NPW, welcher in vorherigen Studien ebenfalls, und dies ausnahmslos, über $95 \%$ lag. Dieser spiegelt das klinische Potential der MDCT in einem Kollektiv mit niedrigem KHK Risiko, wie bei unseren Patienten mit symptomatischem VHF vor Ablationsversuch der Fall, nichtinvasiv und suffizient stenosierte Koronararterien auszuschließen.

\subsection{Koronare Herzkrankheit und Vorhofflimmern}

Die KHK ist ein deutlicher, prädisponierender Faktor für das Auftreten von VHF (Kannel et al. 1982). In diesem Zusammenhang wurden in einer Studie von Nucifora et al. 150

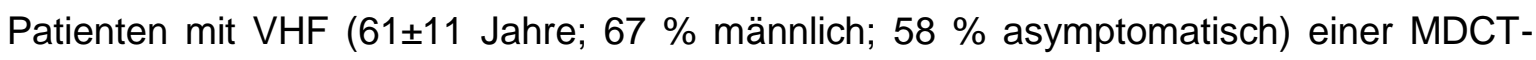
Koronarangiographie unterzogen. Ziel war es, die Prävalenz einer KHK bei diesem Patientenkollektiv zu beziffern. Als Kontrollgruppe wurden 148 Patienten ohne VHF in der Anamnese gewählt. In ersterer Kohorte hatten $18 \%$ der Patienten It. CT keine KHK, während $41 \%$ nicht-stenosierende- (< $50 \%$ Lumeneinengung) und die restlichen $41 \%$ stenosierende- (> 50 \% Lumeneinengung) Charakteristika einer KHK zeigten. Bei der Kontrollgruppe wiesen $32 \%$ keine KHK auf, $41 \%$ der Patienten hatten eine nichtstenosierende- und $27 \%$ eine stenosierende KHK lt. CT. Somit kam die Forschungsgruppe zu dem Schluss, dass bei Patienten mit VHF eine erhöhte Prävalenz für eine obstruktive KHK besteht (Nucifora et al. 2009). In unserer Studie erkannte die MDCT bei $3 \%$ der Patienten eine signifikante KHK und in $21 \%$ eine nicht-signifikante KHK. In der invasiven Koronarangiographie dagegen wurden in $1 \%$ der Fälle signifikante Stenosen und in $17 \%$ der Fälle nicht-signifikante Stenosen diagnostiziert. Unserer Einschätzung nach lassen sich die hohen Werte von Nucifora et al. dadurch erklären, dass keine Vergleichsergebnisse der invasiven Koronarangiographie vorlagen und somit sicherlich von einer hohen Rate an falsch positiven Ergebnissen ausgegangen werden muss. Weiterhin differiert ihr Studiendesign in den Punkten Alter, Geschlecht und Risikofaktoren (Nucifora et al. 2009). 


\subsection{Diagnostischer Nutzen der MDCT}

Laut Angaben des Statistischen Bundesamtes ist die KHK heutzutage die häufigste Todesursache (Gesundheitsberichterstattung des Bundes 2011). Diese Tatsache macht es so interessant, mittels 64-Zeilen-MDCT nichtinavasiv eine signifikante Stenosierung bei Patienten mit niedrigem bis mittlerem Risiko für eine KHK auszuschließen (Budoff und Gul 2008).

Gerade bei unserem Patientenkollektiv scheint dieses Vorgehen eine logische Konsequenz zu sein, da routinemäßig vor PVA eine MDCT des Herzens durchgeführt wird und man somit zwei eigentlich getrennte diagnostische Methoden zusammenlegen kann: Zum einen die Darstellung des linken Atriums und der PV-Ostien, zum anderen die detaillierte Visualisierung der Koronararterien. Damit erhält man zusätzlich wertvolle Informationen über das eventuelle Vorhandensein einer KHK; weiterhin lässt der hohe NPW aller bis dato vorhandenen Studien den Rückschluss zu, dass bei unauffälliger Darstellung der Koronararterien eine invasive Folgeuntersuchung mittels invasiver Koronarangiographie überflüssig ist.

\subsection{MDCT- Koronarangiographie}

\subsubsection{Einflussfaktoren und technische Weiterentwicklung}

Beleuchtet man die wesentlichen Einflussfaktoren der CT-Koronarangiographie, so finden diesbezüglich drei Punkte Erwähnung. Als Erstes ist die schwere Arteriosklerose zu nennen, die in älteren Studien oftmals durch konsekutive Bildartefakte als limitierender Faktor angegeben wurde und zum wiederholten Ausschluss von Segmenten führte (Ropers et al. 2003; Martuscelli et al. 2004). Eine Tatsache, die in aktuellen Studien noch beschrieben wird, sich jedoch durch die immer höhere örtliche Auflösung sowie schnelleren Rotationszeiten der CT mittlerweile relativiert hat (Wehrschuetz et al. 2010). In dieser Arbeit führte diese Variable lediglich zu einer Reduktion der Bildqualität, ein Ausschluss von Segmenten durch entstandene Bildartefakte war jedoch nicht notwendig. Ein weiterer Punkt sind Gefäßstents, die ebenfalls zu Strahlungs- und Volumenartefakten führen können (Kuettner et al. 2004). Dies war auch in unserer Studie der Fall und führte zum Ausschluss von insgesamt 6 Segmenten, die aufgrund überlagernder Artefakte nicht mehr beurteilbar waren. 
Als dritter Einflussfaktor wird oftmals die HF beschrieben (Giesler et al. 2002; Schroeder et al. 2002; Niemann et al. 2002 a). Hier besteht eine inverse Korrelation zu der Bildqualität, eine bestmögliche Beurteilung der Koronararterien wird für Frequenzen < 65 S/min angegeben (Schroeder et al. 2002; Kuettner et al. 2004). Ferencik et al. führten hierzu 2006 eine retrospektive Studie bezüglich der Bildqualität der 64-Zeilen-CT durch und kamen korrespondierend zu vorherigen Studien zu dem Schluss, dass die Höhe der HF den dargestellten Verlauf der Koronararterien ohne Bewegungsartefakte signifikant beeinflusst (Ferencik 2006). Auch in unserem Patientenkollektiv wurde aus diesem Grund ab einer Herzfrequenz > $70 \mathrm{~S} / \mathrm{min}$ ein Betablocker appliziert, so dass die durchschnittliche Frequenz während der MDCT bei $67 \pm 8 \mathrm{~S} / \mathrm{min}$ lag.

Die Güte der bildlichen Darstellung des Herzens hat jedoch dank stetiger Weiterentwicklung und Neuerungen auf diesem Gebiet weiterhin deutlich zugenommen. Hierbei sind vor allem eine höhere örtliche sowie zeitliche Auflösung, bei gleichzeitig geringerer Belichtungszeit, zu nennen. Mittlerweile steht, neben 320-Zeilen-CT, die DualSource-CT-Technologie (DSCT) zur Verfügung, die mit 2 Röntgenröhren arbeitet und hierdurch eine sehr hohe Bildqualität sowie diagnostische Genauigkeit gewährleistet. Johnson und Mitarbeiter erzielten in einer Studie mit dem DSCT eine Sensitivität von $100 \%$ und eine Spezifität von $89 \%$ bei der Beurteilung signifikanter Koronarstenosen. Kritisch betrachtet, entsprechen diese Werte denen mit einem 64-Zeilen-CT, jedoch liegt der Unterschied in dem deutlich geringeren Anteil von nicht verwertbaren KoronararterienSegmenten in der DSCT (Johnson et al. 2007). Weiterhin ermöglicht die DSCT auch bei tachykarden sowie arrhythmischen Herzphasen das Generieren hochwertiger Bilder, so dass die Gabe von Betablockern nicht mehr erforderlich ist (Johnson et al. 2007; Leber et al. 2007; Rixe et al. 2008).

Bezüglich der 320-Zeilen-CT berichten Khan et al. von einer im Vergleich zur 64-ZeilenCT weiter zugenommenen Bildqualität, bei gleichzeitig reduzierter Strahlenexposition. Mittels 320-MDCT waren 96,66 \% der beurteilten Segmente von exzellenter Qualität, lediglich 0,1\% konnten nicht beurteilt werden. In der 64-MDCT dagegen waren $86 \%$ der Segmente von exzellenter Qualität, während 3,33 \% nicht beurteilbar waren (Khan et al. 2011). 


\subsubsection{Strahlenexposition}

Mit zunehmender Verwendung der MDCT etablierte sich die testassoziierte Strahlenexposition in der Vergangenheit als deutlicher Kritikpunkt sowie als entscheidende Limitation dieser Untersuchung. Einstein et al. beschäftigten sich diesbezüglich in einer 2007 von ihnen publizierten Studie mit der Wahrscheinlichkeit, nach Durchführung einer 64-Zeilen-MDCT an einem Malignom zu erkranken. Hierbei wurden die Ergebnisse in Abhängigkeit von Alter, Geschlecht und verwendetem Scan-Protokoll errechnet. Ohne EKG-Gating während der MDCT lag die Wahrscheinlichkeit zu Lebzeiten an einem Malignom zu erkranken, für eine 20-jährige Frau bei 1 zu 143, für einem 80jährigen Mann wurde sie mit 1 zu 3261 angegeben. Unter Verwendung des EKG-Gatings reduzierte sich die Wahrscheinlichkeit für Frauen auf 1 zu 2019 und für Männer auf 1 zu 5017 (Einstein et al. 2007). Grundsätzlich ist bei dem EKG-Gating ein retrospektives von einem prospektivem Verfahren zu unterscheiden. Bei der prospektiven EKG-Triggerung werden die Aufnahmen R-Zacken getriggert und jeweils während der Diastole angefertigt. Im Gegensatz dazu werden beim retrospektiven EKG-Gating kontinuierlich und während der gesamten Untersuchungsdauer Aufnahmen angefertigt. Schließlich können durch Verknüpfung mit den EKG-Signalen, retrospektiv die Bilddaten dem jeweiligen Herzzyklus zugeordnet werden. Somit gelingt mittels prospektivem EKG-Gating eine Minimierung der Strahlenexposition, der Vorteil des retrospektiven EKG-Gatings liegt in einer höheren Bildqualität (Shuman et al. 2008).

Betrachtet man in diesem Zusammenhang die Strahlendosen während einer Aufnahme mit früheren CT-Generationen, so dokumentierten beispielsweise Mollet et al., unter Verwendung eines 16-Zeilen-MDCT, Werte zwischen 6,7 und $13 \mathrm{mSv}$. Die Forschungsgruppe um Moon verglich die effektive Strahlendosis eines 64-Zeilen-MDCT in Abhängigkeit von der HF, dies jedoch unter konsequenter Verwendung des prospektiven EKG-Gatings. Hier waren verglichen mit den MDCT älterer Generation, deutlich niedrigere Werte zu verzeichnen. So lag die Strahlendosis bei HF unter $65 \mathrm{~S} / \mathrm{min}$ bei $5,5 \mathrm{mSv}$ sowie bei 6,7 $\mathrm{mSv}$ bei einer HF über $65 \mathrm{~S} / \mathrm{min}$. Deutlicher differierten die Werte bei unterschiedlicher HF-Variabilität: So lag die Strahlenexposition bei einer Variabilität $<15$ $\mathrm{S} / \mathrm{min}$ bei $5,3 \mathrm{mSv}$, wohingegen bei $>15 \mathrm{~S} / \mathrm{min}$ 9,3 $\mathrm{mSv}$ errechnet wurden (Moon et al. 2011).

Bezüglich der 320-Zeilen-CT berichten Khan et al. von einer im Vergleich zur 64-ZeilenCT weiter zugenommenen Bildqualität, bei gleichzeitig reduzierter Strahlenexposition. In dem Patientenkollektiv mit einer HF $\leq 65 \mathrm{~S} / \mathrm{min}$ lag die mittlere Strahlendosis mit dem 320-Zeilen-CT bei 4,1 mSv, während sie bei dem 64-Zeilen-CT mit 6,2 mSv angegeben wurde (Khan et al. 2011). 
Betrachtet man die einzelnen auf die Strahlendosis einflussnehmenden Faktoren so lassen sich die in den einzelnen Studien teilweise deutlich differierenden Mittelwerte verstehen. Hierbei hilft die Studie Prospective Multicenter Study On Radiation Dose Estimates Of Cardiac CT Angiography In Daily Practice I (PROTECTION I), in der die Daten aus 50 Kliniken verschiedener Länder, die ihrerseits eine entsprechende Expertise mit der CT-Koronarangiografie hatten untersucht wurden. Auffällig ist, dass bei der Strahlenexposition deutlich differierende Werte zwischen 5 und $30 \mathrm{mSv}$ erreicht wurden (Hausleiter et al. 2009). Einflussnehmend waren hier vornehmlich die Röhrenspannung, das EKG-Gating sowie das sequentielle Scannen. Weiterhin waren das Körpergewicht sowie die HF entscheidende Variablen.

Vergleicht man nun die effektive Strahlendosis der MDCT mit der der invasiven Koronarangiographie, so fallen zum jetzigen Zeitpunkt allenfalls geringe Differenzen auf, die sich in Zukunft weiter annähern werden. Eine Studie aus dem Jahre 2006 ergab diesbezüglich in einer Kohorte von 73 Patienten eine Strahlenexposition von 12,3 \pm 1,4 mSv in der 64-Zeilen-CT Koronarangiographie, wohingegen bei der invasiven Koronarangiographie Werte von 11,4 \pm 4,8 mSv erreicht wurden (Dewey et al. 2006). Insgesamt erscheinen die hierbei ermittelten Werte vergleichsweise hoch, eine Erklärung ist bezogen auf die invasive Koronarangiographie, dass neben einem diagnostischen Herzkatheter ebenfalls eine Intervention im Sinne einer PKI mit zusätzlicher Strahlenexposition durchgeführt wurde. Die hohen Werte der MDCT können mit den zuvor bereits erwähnten und einflussnehmenden Faktoren erklärt werden.

In unserem Fall erhielten alle Patienten eine CT zur Darstellung des linken Vorhofes und der PV-Mündungen als Routinemaßnahme vor Ablation, so dass für die MDCTKoronarangiographie keine zusätzliche Strahlenexposition entstand. Neben diesem Vorteil besteht ein weiterer Vorteil darin, dass nach Ausschluss signifikanter Stenosen mittels MDCT eine diagnostische Herzkatheteruntersuchung mit konsekutiver Strahlenexposition überflüssig wurde. 


\subsubsection{Kosteneffektivität der MDCT-Koronarangiographie im Vergleich zur invasiven Koronarangiographie}

Vergleicht man die entstehenden Kosten sowohl der invasiven als auch der nichtinvasiven Koronarangiographie, gestaltet sich die Zusammensetzung der Gesamtkosten wie folgt: Die direkten Kosten der Untersuchung einschließlich Folgeuntersuchung, Kosten der jeweiligen Komplikationen sowie Folgekosten bei der Identifizierung von Patienten als falsch negativ bzw. als falsch positiv.

Das Deutsche Institut für Medizinische Dokumentation und Information (DIMDI) führte in diesem Zusammenhang 2012 eine Literaturrecherche bezüglich der Kosten-Wirksamkeit der CT-Koronarangiographie versus invasiver Koronarangiographie durch. Hierbei wurden lediglich die mit mindestens 64-Zeilen-CT-Geräten erhobenen Daten sowie Publikationen ab 2006 analysiert. Die Autoren kommen zu dem Schluss, dass bei einer Prätestwahrscheinlichkeit von $\leq 50 \%$ für eine $\mathrm{KHK}$, die Kosten der CT-Angiographie deutlich niedriger als die der invasiven Koronarangiographie sind. Dies ist der Tatsche geschuldet, dass bei dieser Prätestwahrscheinlichkeit größtenteils eine stenosierende KHK mittels MDCT ausgeschlossen werden und eine invasive Koronarangiographie als Folgeuntersuchung mit möglicherweise revaskulierenden Maßnahmen verhindert werden kann (Gorenoi et al. 2012).

Zu einem vergleichbaren Ergebnis kam die Forschungsgruppe um Dorenkamp. Auch hier war die Prätestwahrscheinlichkeit eine entscheidende Determinate für die Höhe der Kosten. Die direkten Kosten für die MDCT-Koronarangiographie wurden dort mit 98,60€ bzw. 317,75€ für die invasive Koronarangiographie beziffert. Wurden diese Beträge nun hochgerechnet auf die Gesamtkosten bzgl. der Detektion eines als richtig positiv erkannten Patienten mit einer signifikanten KHK, ergaben sich Werte von 970,00€ für die MDCT sowie 1354,00 € für die invasive Koronarangiographie. Dies galt allerdings erneut nur für eine Prätestwahrscheinlichkeit $\leq 50 \%$. Bei einer höheren Prätestwahrscheinlichkeit verlagerte sich die Kosteneffektivität zu Gunsten der invasiven Koronarangiographie (Dorenkamp et al. 2012).

Eine Arbeitsgruppe aus Madrid beschäftigte sich ebenfalls mit den entstehenden Kosten der einzelnen Untersuchungen, legte in diesem Zusammenhang jedoch den Fokus auf die Ermittlung vermeidbarer Folgekosten bei der Durchführung einer primären MDCT. Anhand eine Analyse von 4 großen europäischen Studien mit insgesamt 490 Patienten kamen sie zu dem Ergebnis, dass 71,2 \% der invasiven Koronarangiographie und 3,56 \% der postinterventionellen Komplikationen durch eine initiale CT-Koronarangiographie hätten vermieden werden können, dies wiederum entspräche einer Einsparung von $411,00 €$ pro Patient (Catalán et al. 2013). 
Diese Daten lassen die Schlussfolgerung zu, dass, bezogen auf die okönomischen Auswirkungen, die Vorschaltung einer MDCT bei Patienten mit niedriger oder mittlerer Wahrscheinlichkeit für das Vorliegen einer signifikanten Koronararterienstenose kosteneffizient bzw. kostensparend ist. Aufgrund eines hohen NPW kann mittels der CTKoronarangiographie eine signifikante KHK ausgeschlossen und eine invasive Untersuchung mit zusätzlichen Kosten verhindert werden.

\subsubsection{Limitationen der MDCT}

Ältere Studien beschreiben schwere Arteriosklerose als limitierenden Faktor, der oftmals zum Ausschluss von Segmenten führt (Ropers et al. 2003; Martuscelli et al. 2004). Eine Erklärung hierfür ist möglicherweise die Tatsache, dass Martuscelli und Ropers lediglich ein 16-Zeilen-CT zur Beurteilung der Koronararterien wählten. Dieser Punkt verdeutlicht jedoch, warum die MDCT-Koronarangiograpie in der Vergangenheit im klinischen Alltag als nicht relevantes Diagnostikum angesehen wurde: Wollte man die Arteriosklerose als Ursache der KHK bewerten, so verhinderte diese in schweren Fällen paradoxerweise die Analyse der entsprechenden Segmente. Eine Tatsache, die in aktuellen Studien noch beschrieben wird, sich jedoch durch die immer besser werdende Auflösung mittlerweile relativiert hat (Wehrschuetz et al. 2010). Auch in unserem Fall stell diese Variable lediglich eine Reduktion der Bildqualität dar, jedoch ohne zum Ausschluss zu führen. Folglich ist dieser Punkt für unsere Studie von keiner diagnostischer Relevanz.

Einen weiteren, limitierenden Faktor stellen Gefäßstents dar, die ebenfalls zu Strahlungsund Volumenartefakten führen können (Kuettner et al. 2004). Dies ist auch in unserer Studie der Fall und führt zum Ausschluss von 6 Segmenten, da in allen Fällen die Gefäße durch überlagernde Artefakte nicht mehr beurteilbar waren. Diesbezüglich hat sich jedoch mittlerweile die diagnostische Güte in der Beurteilung von Koronarartieren mit einliegenden Stents aufgrund weiter entwickelter Rekonstruktions- und Bildbearbeitungsalgorithmen deutlich verbessert.

Des Weiteren ist die fehlende Möglichkeit einer Intervention, wie sie etwa bei der invasiven Koronarangiographie im Sinne einer PKI mit oder ohne Stentimplantation gegeben ist, eine Einschränkung der MDCT. Doch muss auch dieser Punkt vor dem 
Hintergrund relativiert werden, dass lediglich auf $40 \%$ der invasiven Koronarangiographien eine anschließende Intervention erfolgt (Lehmkuhl et al. 2009).

Eine letzte Einschränkung stellt die oftmals schlechte Beurteilbarkeit einiger weniger Koronararteriensegmente dar. Entsprechend unseren Ergebnissen berichteten Mollet et al., dass ein Drittel der Läsionen > $50 \%$, die mittels MDCT nicht erkannt wurden, in der RCX und ein weiteres Drittel in den kleinen Seitenästen der Koronararterien lokalisiert waren. Diese Tatsache stellt eine Limitation der CT-Koronarangiographie dar und kann durch den geschlängelten Verlauf bzw. überlappende Strukturen erklärt werden (Mollet et al. 2004; Leschka et al. 2005). 


\section{Zusammenfassung}

In der Vergangenheit hat sich die MDCT durch eine stetige Weiterentwicklung der Technik als Verfahren in der nichtinvasiven kardialen Diagnostik, insbesondere der Darstellung der Koronararterien bei Patienten mit niedrigem bis mittlerem Risiko für das Vorliegen einer KHK etabliert.

Das Hauptziel der vorliegenden Arbeit war es, den derzeitigen Stellenwert der 64-ZeilenCT zur Beurteilung von hämodynamisch relevanten Koronarstenosen bei denjenigen Patienten zu evaluieren, die aufgrund eines therapierefraktären, symptomatischen VHF eine Katheterablation erhalten sollten. Als Referenzmethode diente hierbei die konventionelle invasive Koronarangiographie, welche in Abhängigkeit von der PrätestWahrscheinlichkeit für eine KHK durchgeführt wurde. In unserem Patientenkollektiv wurde zwischen einer niedrigen ( $1 \%$ bis $30 \%$ ), einer mittleren (31\% bis $70 \%$ ) und einer hohen (71\% bis $99 \%$ ) Wahrscheinlichkeit für das Vorliegen einer signifikanten Koronarstenose unterschieden. Folglich wurde bei 86 (47\%) Patienten mit mittlerem Risiko jeweils eine MDCT und eine invasive Koronarangiographie durchgeführt. Dagegen erhielten 98 (53\%) Patienten, die keine AP-Beschwerden oder AP-Äquivalente sowie ein niedriges Risiko aufwiesen, lediglich eine MDCT ohne Durchführung einer invasiven Koronarangiographie. Zusammenfassend konnten mittels MDCT-Koronarangiographie 829 von 844 (98\%) Koronarsegmenten, die laut invasiver Koronarangiographie keine Stenosen aufwiesen, auch als solche korrekt detektiert werden. Weiterhin wurden alle Patienten mit einer signifikanten Stenose in einem oder mehreren Segmenten auch als solche richtig erkannt. Bezogen auf die Detektion der in der invasiven Koronarangiographie dokumentierten Stenosen der Koronararterien > $50 \%$, lag die Sensitivität der MDCT bei $90 \%$, die Spezifität bei $98 \%$, der PPW bei $38 \%$ sowie der NPW bei $100 \%$.

Die Ergebnisse unserer Arbeit lassen erkennen, dass die Stärke der CTKoronarangiographie in dem zuverlässigen Ausschluss einer relevanten Koronararterienstenose bei Patienten mit niedriger bis mittlerer Wahrscheinlichkeit für das Vorliegen einer KHK liegt. Mittels der routinemäßig vor PVA generierten Bilddaten der 64-Zeilen-MDCT können neben der für die Ablation wichtigen LA-Anatomie die klinisch bedeutsamen Abschnitte des Koronarbaumes in einem Schritt mit visualisiert werden. Dies ermöglicht die Minimierung der Strahlenexposition und der Kosten sowie das Vermeiden der mit invasiver Koronarangiographie assoziierten Morbidität und Mortalität: Somit kann bei negativem Befund in der MDCT auf eine invasive Folgeuntersuchung mittels Herzkatheter verzichtet werden. 
Die klinische Etablierung neuerer und technisch weiterentwickelter MDCT-Systeme, wie zum Beispiel die Einführung der Dual-Source-Technologie oder die Entwicklung eines 320-Zeilen-CT-Gerätes sowie die Verbesserung der Scan-Protokolle führen sowohl zu einer weiteren Reduktion der Strahlenexposition als auch zu einem erweiterten Einsatz der CT-Koronarangiographie. 


\section{Literaturverzeichnis}

Achenbach S, Ulzheimer S, Baum U, Kachelriess M, Ropers D, Giesler T, Bautz W, Daniel WG, Kalender WA, Moshage W (2000): Noninvasive coronary angiography by retrospectively ECG-gated multislice spiral CT. Circulation 102, 2823-2828

Achenbach S, Giesler T, Ropers D, Ulzheimer S, Derlien H, Schulte C, Wenkel E, Moshage W, Bautz W, Daniel G (2001): Detection of coronary artery stenoses by contrast-enhanced, retrospectively electrocardiographically-gated, multislice spiral computed tomography. Circulation $\underline{103}, 2535-2538$

Alpert JS, Petersen P, Godtfredsen J (1988): Atrial fibrillation: natural history, complications, and management. Annu Rev Med $\underline{39}, 41$

Altman D: Practical Statistics for Medical Research. Chapman \& Hall Verlag, London 1991

Arentz T, Jander N, von Rosenthal J, Blum T, Fürmaier R, Görnandt L, Josef Neumann F, Kalusche D (2003): Incidence of pulmonary vein stenosis 2 years after radiofrequency catheter ablation of refractory atrial fibrillation. Eur Heart J 24, 963-969

Austen WG, Edwards JE, Frye RL, Gensini GG, Gott VL, Griffith LS, McGoon DC, Murphy $M L$, Roe BB (1975): A reporting system on patients evaluated for coronary artery disease. Report of the Ad Hoc Committee for Grading of Coronary Artery Disease, Council on Cardiovascular Surgery, American Heart Association. Circulation 51, 5-40

Becker CR, Knez A, Leber A, Hong C, Treede H, Wildhirt S, Ohnesorge B, Flohr T, Schoepf UJ, Reiser MF (2000): CT in der Diagnostik der Arteriosklerose der Koronargefäße. Radiologe $\underline{40}, 118-122$

Benjamin EJ, Levy D, Vaziri SM, D'Agostino RB, Belanger AJ, Wolf PA (1994): Independent risk factors for atrial fibrillation in a population-based cohort. The Framingham Heart Study. JAMA 271, 840-844

Bestehorn H-P: Interventionelle Kardiologie - Koronarangiographie und PTCA Indikation, Technik, Nachsorge. 2. Auflage; Thieme, Stuttgart, 2001 
Blomstrom-Lundqvist C, Scheinman MM, Aliot EM, Alpert JS, Calkins H, Camm AJ, Campbell WB, Haines DE, Kuck KH, Lerman BB; American College of Cardiology; American Heart Association Task Force on Practice Guidelines; European Society of Cardiology Committee for Practice Guidelines. Writing Committee to Develop Guidelines for the Man-agement of Patients With Supraventricular Arrhythmias (2003): ACC/AHA/ESC guidelines for the management of patients with supraventricular arrhythmias--executive summary: a report of the American College of Cardiology/American Heart Association Task Force on Practice Guidelines and the European Society of Cardiology Committee for Practice Guidelines (Writing Committee to Develop Guidelines for the Management of Patients With Supraventricular Arrhythmias). Circulation 108, 1871-1909

Brand FN, Abbott RD, Kannel WB, Wolf PA (1985): Characteristics and prognosis of lone atrial fibrillation. 30-year follow-up in the Framingham Study. JAMA 254, 3449-3453

Budoff MJ, Gul KM (2008): Expert review on coronary calcium. Vasc Health Risk Manag 4, 315-324

Camm AJ, Kirchhof P, Lip GY, Schotten U, Savelieva I, Ernst S, Van Gelder IC, Al-Attar N, Hindricks G, Prendergast B (2010): Guidelines for the management of atrial fibrillation: the Task Force for the Management of Atrial Fibrillation of the European Society of Cardiology (ESC). Eur Heart J $\underline{31}$, 2369-2429

Camm AJ, Lip GY, De Caterina R, Savelieva I, Atar D, Hohnloser SH, Hindricks G, Kirchhof P; ESC Committee for Practice Guidelines(CPG) (2012): 2012 focused update of the ESC Guidelines for the management of atrial fibrillation: anupdate of the 2010 ESC Guidelines for the management of atrial fibrillation. Developed with the special contribution of the European Heart Rhythm Association. Eur Heart J 33, 2719-4277

Catalán P, Callejo D, Blasco JA (2013): Costeffectiveness analysis of 64-slice computed tomography vs cardiac catheterization to rule out coronary artery disease before noncoronary cardiovascular surgery. Eur Heart J Cardiovasc Imaging 14, 149-157

Cheema A, Dong J, Dalal D, Marine J, Henrikson C, Spragg D, Cheng A, Nazarian S, Bilchick K, Almasry I (2007): Circumferential Ablation With Pulmonary Vein Isolation in Permanent Atrial Fibrillation. Am J Cardiol 99, 1425-1428 
Chen SA, Hsieh MH, Tai CT, Tsai CF, Prakash VS, Yu WC, Hsu TL, Ding YA, Chang S (1999): Initiation of atrial fibrillation by ectopic beats originating from the pulmonary veins: electrophysiological characteristics, pharmacological responses, and effects of radiofrequency ablation. Circulation $\underline{100}, 1879-1886$

Cohen EJ, Klatsky AL, Armstrong MA (1988): Alcohol use and supraventricular arrhythmia. Am J Cardiol $\underline{62}, 971$

Cox JL, Canavan TE, Schuessler RB, Cain ME, Lindsay BD, Stone C, Smith PK, Corr PB, Boineau JP (1991 a): The surgical treatment of atrial fibrillation. II. Intraoperative electrophysiologic mapping and description of the electrophysiologic basis of atrial flutter and atrial fibrillation. J Thorac Cardiovasc Surg $\underline{101}$, 406-426

Cox JL, Schuessler RB, D'Agostino HJ Jr, Stone CM, Chang BC, Cain ME, Corr PB, Boineau JP (1991 b): The surgical treatment of atrial fibrillation. III. Development of a definitive surgical procedure. J Thorac Cardiovasc Surg 101, 569-583

De Haen C (1995): Gebührenordnung für Ärzte (GOÄ) vom 12. November 1982 (BGBI.IS.1522) zuletzt geändert durch die 4. Änderungsverordnung von 23. Dezember 1995 (BGBI. IS.1861 ff)

Deisenhofer I, Estner H, Luik A, Pflaumer A, Pustowoit A, Zrenner B, Schmitt C (2006): Die Integration von individuellen anatomischen 3D-Rekonstruktionen aus MehrschichtComputertomographien in das elektro-anatomische CartoMerge ${ }^{\circledR}$ System bei der Radiofrequenzablation von paroxysmalem und persistierendem Vorhofflimmern. Clin Res Cardiol $\underline{95}$, Suppl 5

Delhaye D, Remy-Jardin M, Salem R, Teisseire A, Khalil C, Delannoy-Deken V, Duhamel A, Remy J (2007): Coronary imaging quality in routine ECG-gated multidetector CT examinations of the entire thorax: preliminary experience with a 64-slice CT system in 133 patients. Eur Radiol 17, 902 - 910

Dewey M, Teige F, Schnapauff D, Laule M, Borges AC, Wernecke KD, Schink T, Baumann G, Rutsch W, Rogalla P (2006): Noninvasive Detection of Coronary Artery Stenoses with Multislice Computed Tomography or Magnetic Resonance Imaging. Ann Intern Med $\underline{145}$, 407-415 
Dorenkamp M, Bonaventura K, Sohns C, Becker CR, Leber AW (2012): Direct costs and cost-effectiveness of dual-source computed tomography and invasive coronary angiography in patients with an intermediate pretest likelihood for coronary artery disease. Heart $\underline{98}, 460-467$

Einstein AJ, Henzlova MJ, Rajagopalan M (2007): Estimation of risk of cancer associated with radiation exposure from 64-slice computed tomography coronary angiography. JAMA $\underline{298}, 317-323$

Eldar M, Canetti M, Rotstein Z, Boyko V, Gottlieb S, Kaplinsky E, Behar S (1998): Significance of paroxysmal atrial fibrillation complicating acute myocardial infarction in the thrombolytic era. SPRINT and thrombolytic survey groups. Circulation $\underline{97}, 965-970$

Ernst S, Schlüter M, Ouyang F, Khanedani A, Cappato R, Hebe J, Volkmer M, Antz M, Kuck KH (1999): Modification of the substrate for maintenance of idiopathic human atrial fibrillation: efficacy of radiofrequency ablation using nonfluoroscopic catheter guidance. Circulation 100, 2085-2092

Ferencik M, Nomura CH, Maurovich-Horvat P, Hoffmann U, Pena AJ, Cury RC, Abbara S, Nieman K, Fatima U, Achenbach S (2006): Quantitative parameters of image quality in 64-slice computed tomography angiography of the coronary arteries. Eur J Radiol 57, 373-379

Furberg CD, Psaty BM, Manolio TA, Gardin JM, Smith VE, Rautaharju PM (1994): Prevalence of atrial fibrillation in elderly subjects (the Cardiovascular Health Study). Am J Cardiol $\underline{74}, 236-241$

Fuster V, Rydén LE, Cannom DS, Crijns HJ, Curtis AB, Ellenbogen KA, Halperin JL, Le Heuzey JY, Kay GN, Lowe JE (2006): ACC/AHA/ESC 2006 Guidelines for the Management of Patients with Atrial Fibrillation: a report of the American College of Cardiology/American Heart Association Task Force on Practice Guidelines and the European Society of Cardiology Committee for Practice Guidelines (Writing Committee to Revise the 2001 Guidelines for the Management of Patients With Atrial Fibrillation): developed in collaboration with the European Heart Rhythm Association and the Heart Rhythm Society. Circulation $\underline{114}$, 257-354 
Gepstein L, Hayam G, Ben Haim SA (1997): A novel method for nonfluoroscopic catheterbased electroanatomical mapping of the heart. In vitro and in vivo accuracy results. Circulation $\underline{95}, 1611-1622$

Gerber B (2009): Comprehensive cardiac imaging using multidetector CT and magnetic resonance imaging. Bull Mem Acad R Med Belg $\underline{164}$, 103-108

Gerstenfeld EP, Guerra P, Sparks PB, Hattori K, Lesh MD (2001): Clinical outcome after radiofrequency catheter ablation of focal atrial fibrillation triggers. $J$ Cardiovasc Electrophysiol $\underline{12}, 900-908$

Gibbons RJ, Balady GJ, Bricker JT, Chaitman BR, Fletcher GF, Froelicher VF, Mark DB, McCallister BD, Mooss AN, O'Reilly MG (2002): Noninvasive visualisation of coronary arteries using contrast-enhanced multidetector CT: influence of heart rate on image quality and stenosis detection. Am J Roentgenol $\underline{179}, 911-916$

Giesler T, Baum U, Ropers D, Ulzheimer S, Wenkel E, Mennicke M, Bautz W, Kalender WA, Daniel WG, Achenbach S (2002). Noninvasive visualisation of coronary arteries using contrast-enhanced multidetector CT: influence of heart rate on image quality and stenosis detection. Am J Roentgenol 179, 911-916

Gorenoi V, Schönermark MP, Hagen A (2012): CT-Koronarangiografie versus konventionelle invasive Koronarangiografie bei der KHK-Diagnostik. DIMDI 117

Haissaguerre M, Marcus FI, Fischer B, Clementy J (1994): Radiofrequency catheter ablation in unusual mechanisms of atrial fibrillation: report of three cases. $\mathrm{J}$ Cardiovasc Electrophysiol $\underline{5}, 743-751$

Haissaguerre M, Jais P, Shah DC, Takahashi A, Hocini M, Quiniou G, Garrique S, Le Mouroux A, Le Metayer P, Clementy J (1998): Spontaneous initiation of atrial fibrillation by ectopic beats originating in the pulmonary veins. N Engl J Med $\underline{339}, 659-666$

Haissaguerre M, Jais P, Shah DC, Garrigue S, Takahashi A, Lavergne T, Hocini M, Peng JT, Roudaut R, Clementy J (2000 a): Electrophysiological end point for catheter ablation of atrial fibrillation initiated from multiple pulmonary venous foci. Circulation $\underline{10}, 1409$ 1417 
Haissaguerre $M$, Shah DC, Jais $P$, Hocini $M$, Yamane $T$, Deisenhofer I, Chauvin $M$, Garrigue S, Clementy J (2000 b): Electrophysiological breakthroughs from the left atrium to the pulmonary veins. Circulation 102, 2463-2465

Hamoir XL, Flohr T, Hamoir V, Labaki L, Tricquet JY, Duhamel A, Kirsch J (2005): Coronary arteries: assessment of image quality and optimal reconstruction window in retrospective ECG gated multislice CT at 375 ms gantry rotation time. Eur Radiol $\underline{15}, 296$ $-304$

Hannink M, Laubinger G (1982): Das klinische Bild des idiopathischen paroxysmalen Vorhofflimmerns. Herz/Kreislauf $\underline{8}, 446$

Hausleiter J, Meyer T, Hermann F, Hadamitzky M, Krebs M, Gerber TC, McCollough C, Martinoff S, Kastrati A, Schömig A (2009): Estimated Radiation Dose Associated With Cardiac CT Angiography. JAMA 301, 500-507

Heeringa J, van der Kuip DA, Hofman A, Kors JA, van Herpen G, Stricker BH, Stijnen T, Lipet GY, Witteman JC (2006): Prevalence, incidence and lifetime risk of atrial fibrillation: the Rotterdam study. Eur Heart 27, 949- 953

Herzog C, Abolmaali N, Balzer JO, Baunach S, Ackermann H, Dogan S, Britten MB, Vogl TJ (2002): Heart-rate-adapted image reconstruction in multidetector-row cardiac CT: influence of physiological and technical prerequisite on image quality. Eur Radiol $\underline{12}$, 2670-2678

Hindricks G, Kottkamp H (2001): Simultaneous noncontact mapping of left atrium in patients with paroxysmal atrial fibrillation. Circulation 104, 297- 303

Hoffmann MH, Shi H, Johnson PC, Aschoff AJ (2004): Nichtinvasive Koronarangiographie mittels Mehrschicht-CT: Klinische Anwendungen. Medica Mundi $\underline{6}$, 45-50

Incalzi RA, Postelli R, Fuso L, Cocchi A, Bonetti MG, Giordano A (1990): Cardiac arrhythmias and left ventricular function in respiratory failure from chronic obstructive pulmonary disease. Chest $\underline{97}, 1092-1097$ 
Jais P, Shah DC, Haissaguerre M, Takahashi A, Lavergne T, Hocini M, Garrigue S, Barold SS, Le Metayer P, Clementy J (1999): Efficacy and safety of septal and left-atrial linear ablation for atrial fibrillation. Am J Cardiol $\underline{84}, 139 \mathrm{R}-146 \mathrm{R}$

Johnson TR, Nikolaou K, Busch S, Leber AW, Becker A, Wintersperger BJ, Rist C, Knez A, Reiser MF, Becker CR (2007): Diagnostic accuracy of dual-source computed tomography in the diagnosis of coronary artery disease. Invest Radiol. $\underline{42}, 684-91$

Kalender WA (1999): Grundlagen und Technik der Spiral-CT. Radiologe 39, 809-819

Kannel WB, Abbott RD, Savage DD, McNamara PM (1982): Epidemiologic features of chronic atrial fibrillation: the Framingham study. N Engl J Med $\underline{306}, 1018-22$

Khan A, Khosa F, Nasir K, Yassin A, Clouse ME (2011): Comparison of radiation dose and image quality: 320-MDCT versus64-MDCT coronary angiography. Am J Roentgenol 197, 163-168

Khargi K, Hutten BA, Lemke B, Deneke T (2005): Surgical treatment of atrial fibrillation; a systematic review. Eur J Cardiothorac Surg 27, 258-265

Knaut M, Tugtekin SM, Jung F, Matschke K (2004): Microwave ablation for the surgical treatment of permanent atrial fibrillation--a single centre experience. Eur $\mathrm{J}$ Cardiothorac Surg $\underline{26}, 742-746$

Knaut M, Brose S, Tugtekin SM, Kappert U, Jung F, Matschke K (2007): Microwave ablation of permanent atrial fibrillation during isolated bypass grafting and isolated mitral valve surgery. Heart Surg Forum 10, 153-157

Kobelt H, Steinhausen D: Wirtschaftsstatistik für Studium und Praxis. 7. Auflage; SchäfferPoeschl Verlag, Stuttgart 2006

Koistinen J, Alin M, Vilkki V, Savola J (2013): Thoracoscopic ablation of atrial fibrillation. Duodecim $\underline{129}$, 57-63

Kopp AF, Schroeder S, Kuettner A, Baumbach A, Georg C, Kuzo R, Heuschmid M, Ohnesorge B, Karsch KR, Claussen CD (2002): Non-invasive coronary angiography with resolution multidetector-row computed tomography. Results in 102 patients. European Heart Journal $\underline{23}, 1714-1725$ 
Kuettner A, Kopp AF, Schroeder S, Rieger T, Brunn J, Meisner C, Heuschmid M, Trabold T, Burgstahler C, Martensen J (2004): Diagnostic accuracy of multidetector computed tomography coronary angiography in patients with angiographically proven coronary artery dise ase. J Am Coll Cardiol $\underline{43}$, 840-841

Kumamaru KK, Arai T, Morita H, Sekine T, Takamura K, Takase S, Rybicki FJ, Kondo T (2014): Overestimation of pretest probability of coronary artery disease by Duke clinical score in patients undergoing coronary CT angiography in a Japanese population. $J$ Cardiovasc Comput Tomogr 8, 198-204

Leber AW, Knez A, Becker C, Becker A, White C, Thilo C, Reiser M, Haberl R, Steinbeck $G$ (2003): Non-invasive intravenous coronary angiography using electron beam tomography and multislice computed tomography. Heart $\underline{89}$, 633-639

Leber AW, Knez A, Becker A, Becker C, von Ziegler F, Nikolaou K, Rist C, Reiser M, White C, Steinbeck G (2004): Accuracy of multidetector spiral computed tomography in identifying and differentiating the composition of coronary atherosclerotic plaques: a comparative study with intracoronary ultrasound. J Am Coll Cardiol $\underline{43}, 1241-1247$

Leber AW, Knez A, von Ziegler F, Becker A, Nicolaou K, Paul S, Wintersperger B, Reiser M, Becker CR, Steinbeck G (2005): Quantification of obstructive and nonobstuctive coronary lesions by 64-slice computed tomography. J Am Coll Cardiol 46, 147-154

Leber AW, Johnson T, Becker A, von Ziegler F, Tittus J, Nikolaou K, Reiser M, Steinbeck G, Becker CR, Knez A (2007). Diagnostic accuracy of dual-source multi-slice CT-coronary angiography in patients with an intermediate pretest like- lihood for coronary artery disease. Eur Heart J $\underline{28}, 2354-2360$

Lehmkuhl L, Grothoff M, Nitzsche S, Thiele H, Schuler G, Mohr FW, Gutberlet M (2009): Computertomographie des Herzens- Dtsch Med Wochenschr 134, 993-997

Leschka S, Alkadhi H, Plass A, Desbiolles L, Grünenfelder J, Marincek B, Wildermuth S (2005): Accuracy of MSCT coronary angiography with 64-slice technology; first experience. Eur Heart J $\underline{26}, 1482-1487$ 
Lin WS, Prakash VS, Tai CT, Hsieh MH, Tsai CF, Yu WC, Lin YK, Ding YA, Chang MS, Chen SA (2000): Pulmonary vein morphology in patients with paroxysmal atrial fibrillation initiated by ectopic beats originating from the pulmonary veins: implications for catheter ablation. Circulation 101,1274-1281

Mantovan R, Verlato R, Calzolari V, Baccillieri S, De Leo A, Turrini P, Pastore G, Crosato M, Ramondo A, Stritoni P (2005): Comparison between anatomical and integrated approaches to atrial fibrillation ablation: adjunctive role of electrical pulmonary vein disconnection. J Cardiovasc Electrophysiol 16, 1293-1297

Martinez MW, Kirsch J, Williamson EE, Syed IS, Feng D, Ommen S, Packer DL, Brady PA 2009: Utility of nongated multidetector computed tomography for detection of left atrial thrombusev in patients undergoing catheter ablation of atrial fibrillation. JACC CardiovasC Imaging 2, 69-76

Martuscelli E, Romagnoli A, D'Eliseo A, Razzini C, Tomassini M, Sperandio M, Simonetti G, Romeo F (2004): Accuracy of thin-slice computed tomography in the detection of coronary stenoses. Eur Heart J $\underline{25}, 1043-1048$

McKenna W, Deanfield J, Farugi A, England D, Oakley C, Goodwin J (1981): Prognosis in hypertrophic cardiomyopathy: role of age and clinical, electrocardiographic and hemodynamic features. Am J Cardiol 쓰, 532-538

Meijboom WB, Nico RM, Carlos AG, Mieghem CAG, Kluin J, Weustink AC, Pugliese F, Vourvouri E, Cademartiri F, Bogers JJC (2006): Pre-Operative Computed Tomography Coronary Angiography to Detect Significant Coronary Artery Disease in Patients Referred for Cardiac Valve Surgery. J Am Coll Cardiol $\underline{48}, 1658$

Moe GK, Abildskov JA (1959). Atrial fibrillation as a self sustaining arrhythmia independent of focal discharge. Am Heart J $\underline{58}, 59-70$

Mollet NR, Cademartiri F, Nieman K, Saia F, Lemos PA, McFadden EP, Pattynama PM, Serruys PW, Krestin GP, de Feyter PJ (2004): Multislice spiral computed tomography coronary angiography in patients with stable angina pectoris. J Am Coll Cardiol 43, 226570 
Montalescot G, Sechtem U, Achenbach S, Andreotti F, Arden C, Budaj A, Bugiardini R, Crea F, Cuisset T, Mario CD (2013): 2013 ESC guidelines on the management of stable coronary artery disease. The Task Force on the management of stable coronary artery disease of the European Society of Cardiology. Eur Heart J $\underline{34}, 2949-3003$

Moon JH, Park EA, Lee W, Yin YH, Chung JW, Park JH, Lee HY, Kang HJ, Kim HY (2011): The Diagnostic Accuracy, Image Quality and Radiation Dose of 64-Slice DualSource CT in Daily Practice: a Single Institution's Experience. Korean J Radiol 12, 308318

Morillo CA, Klein GJ, Jones DL, Guiraudon CM (1995): Chronic rapid atrial pacing. Structural, functional, and electrophysiological characteristics of a new model of sustained atrial fibrillation. Circulation $\underline{91}, 1588-1595$

Morris DC, Hurst JW (1980): Atrial fibrillation. Curr Probl Cardiol $\underline{5}, 5-50$

Narayan SM, Cain ME, Smith JM (1997): Atrial fibrillation. Lancet 350, 943-950

Narayan SM, Kazi D, Krummen DE, Rappel WJ (2008): Repolarization and activation restitution near human pulmonary veins and atrial fibrillation initiation: a mechanism for the initiation of atrial fibrillation by premature beats. J Am Coll Cardiol 52, 1222-1230

Nieman K, Oudkerk M, Rensing BJ, van Ooijen P, Munne A, van Geuns RJ, de Feyter PJ (2001): Coronary angiography with multi-slice computed tomography. Lancet $\underline{357}$, 599603

Nieman K, Rensing B, van Geuns R (2002 a): Non-invasive coronary angiography with multislice spiral computed tomograhy: impact of heart rate. Heart $\underline{88}, 470-474$

Nieman K, Cademartiri F, Lemos PA, Raaijmakers R, Pattynama PM, de Feyter PJ (2002 b): Reliable noninvasive coronary angiography with fast submillimeter multislice spiral computed tomography. Circulation 106, 2051-2054 
Nucifora G, Schuijf JD, Tops LF, van Werkhoven JM, Kajander S, Jukema JW, Schreur JH, Heijenbrok MW, Trines SA, Gaemperli O (2009): Prevalence of coronary artery disease assessed by multislice computed tomography coronary angiography in patients with paroxysmal or persistent atrial fibrillation. Circ Cardiovasc Imaging 2, 100-106

Ohnesorge B, Flohr T, Schaller S, Klingenbeck-Regn K, Becker C, Schöpf UJ, Brüning R, Olgin JE, Zipes DP (2005): Specific Arrhythmias: Diagnosis and Treatment. Braunwald's Heart Disease. 7. Auflage; Elsevier Saunders, Philadelphia, PA, USA, 816-819

Oncel D, Oncel G, Tastan A (2007): Effectiveness of dual-source CT coronary angiography for the evaluation of coronary artery disease in patients with atrial fibrillation: initial experience. Radiology $\underline{245}$, 703-711

Oral H, Knight BP, Tada H, Ozaydin M, Chugh A, Hassan S, Scharf C, Lai SW, Greenstein R, Pelosi F Jr (2002 a): Pulmonary vein isolation for paroxysmal and persistent atrial fibrillation. Circulation 105, 1077-1081

Oral H, Knight BP, Ozaydin M, Chugh A, Lai SW, Scharf C, Hassan S, Greenstein R, Han JD, Pelosi F Jr (2002 b): Segmental ostial ablation to isolate the pulmonary veins during atrial fibrillation: feasibility and mechanistic insights. Circulation 106, 1256-1262

Pappone C, Oreto G, Lamberti F, Vicedomini G, Loricchio ML, Shpun S, Rillo M, Calabro MP, Conversano A, Ben-Haim SA (1999): Catheter ablation of paroxysmal atrial fibrillation using a 3D mapping system. Circulation $\underline{100}, 1203-1208$

Pappone C, Rosanio S, Oreto G, Tocchi M, Gugliotta F, Vicedomini G, Salvati A, Dicandia C, Mazzone P, Santinelli V (2000): Circumferential radiofrequency ablation of pulmonary vein ostia: A new anatomic approach for curing atrial fibrillation. Circulation $\underline{102}, 2619$ 2628

Patwardhan AM, Dave HH, Tamhane AA, Pandit SP, Dalvi BV, Golam K, Kaul A, Chaukar AP (1997): Intraoperative radiofrequency microbipolar coagulation to replace incisions of maze III procedure for correcting atrial fibrillation in patients with rheumatic valvular disease. Eur J Cardiothorac Surg 12, 627-633 
Pouleur AC, le Polain de Waroux JB, Kefer J, Pasquet A, Vanoverschelde JL, Gerber $B L$ (2008): Direct comparison of hole-heart navigator-gated magnetic resonance coronary angiography and 40- and 64-slice multidetector row computed tomography to detect the coronary artery stenosis in patients scheduled for conventional coronary angiography. Circ Cardiovasc Imaging 1, $114-121$

Pryor DB, Shaw L, McCants CB, Lee KL, Mark DB, Harrell FE Jr, Muhlbaier LH Califf RM (1993): Value of the history and physical in identifying patients at increased risk for coronary artery disease. Ann Intern Med 118, 81-90

Raff GL, Gallagher MJ, O'Neill WW, Goldstein JA (2005): Diagnastic accuracy of noninvasive coronary angigraphy using 64-slice spiral computed tomography. J Am Coll Cardiol $\underline{46}, 552-557$

Rixe J, Rolf A, Conradi G, Elsaesser A, Moellmann H, Nef HM, Bachmann G, Hamm CW, Dill T (2008): Image quality on dual-source computed- tomographic coronary angiography. Eur Radiol 18, 1857-1862

Robert Koch-Institut (2011): Gesundheitsberichterstattung des Bundes 2011 (www.gbebund.de)

Ropers D, Baum U, Pohle K, Anders K, Ulzheimer S, Ohnesorge B, Schlundt C, Bautz W, Daniel W, Achenbach S (2003): Detection of Coronary Artery Stenoses With Thin-Slice Multi-Detector Row Spiral Computed Tomography and Multiplanar Reconstruction. Circulation $\underline{107}$, 664-666

Rotter M, Takahashi Y, Sanders P, Haissaguerre M, Jais P, Hsu LF, Sacher F, Pasquié JL, Clementy J, Hocini M (2005): Reduction of fluoroscopy exposure and procedure duration during ablation of atrial fibrillation using a novel anatomical navigation system. Eur Heart J $\underline{26}, 1415-1421$

Ru San T, Chan MY, Wee Siong T, Kok Foo T, Kheng Siang N, Lee SH, Chi Keong C. (2012): Stroke prevention in atrial fibrillation: understanding the new oral anticoagulants dabigatran, rivaroxaban, and apixaban. Thrombosis 108983. Epub 2012 Sep 10. 
Rubinshtein R, Halon DA, Gaspar T, Jaffe R, Karkabi B, Flugelman MY, Kogan A, Shapira R, Peled N, Lewis BS (2007): Usefulness of 64-slice cardiac computed tomographic angiography for diagnosing acute coronary syndromes and predicting clinical outcome in emergency department patients with chest pain of uncertain origin Circulation $\underline{115}, 1762-$ 1768

Saad EB, Rossillo A, Saad CP, Martin DO, Bhargava M, Erciyes D, Bash D, WilliamsAndrews M, Beheiry S, Marrouche NF (2003): Pulmonary vein stenosis after radiofrequency ablation of atrial fibrillation: functional characterization, evolution, and influence of the ablation strategy. Circulation $\underline{108}, 3102-3107$

Schoepf UJ, Becker CR, Ohnesorge BM, Yucel EK (2004): CT of coronary artery disease. Radiology 232, 18-37

Schroeder S, Kopp AF, Kuettner A, Bugstahler C, Herdeg C, Heuschmid M, Baumbach A, Claussen CD, Karsch KR, Seipel L (2002): Influence of heart rate on vessl visibility in noninvasive soronary angiography using new multislice computed to 94 patients. Clin Imaging 26, 106-111

Shuman WP, Branch KR, May JM, Mitsumori LM, Lockhart DW, Dubinsky TJ, Warren BH, Caldwell JH (2008): Prospective versus retrospective ECG gating for 64-detector CT of the coronary arteries: comparison of image quality and patient radiation dose Radiology $\underline{248}, 431-437$

Selzer A, Katayama F (1972): Mitral regurgitation - clinical patterns, pathophysiology, and natural history. Medicine $\underline{51}, 337$

Sibley C, Bluemke D (2009): Will 3.0-T make coronary magnetic resonance angiography competetive with computed tomography angiography? J Am Coll Cardiol $\underline{54}, 77-78$

Skanes AC, Mandapati R, Berenfeld O, Davidenko JM, Jalife J (1998): Spatiotemporal Periodicity During Atrial Fibrillation in the Isolated Sheep Heart. Circulation 98, 1236-1248

Sohns C, Vollmann D, Luethje L, Dorenkamp M, Seegers J, Schmitto J, Zabel M, Obenauer S 2011: MDCT in the diagnostic algorithm in patients with symptomatic atrial fibrillation. World J Radiol $\underline{3}$, 41-46 
Sohns C, Sohns JM, Bergau L, Sossalla S, Vollmann D, Lüthje L, Staab W, Dorenkamp M, Harrison JL, O'Neill MD (2013): Pulmonary vein anatomy predicts freedom from atrial fibrillation using remote magnetic navigation for circumferential pulmonary vein ablation. Eur Heart J Cardiovasc Imaging 14, 684-691

Stein P, Yaekoub A, Matta F, Sostman H (2008): 64-slice CT for diagnosis of coronary artery disease: a systematic review. Am J Med $\underline{121}, 715-725$

Sueda T, Nagata H, Shikata H, Orihashi K, Morita S, Sueshiro M, Okada K, Matsuura Y (1996): Simple left atrial procedure for chronic atrial fibrillation in patients with isolated mitral valve disease. Ann Thorac Surg $\underline{62}, 1796-1800$

Swartz JF, Pellersels G, Silvers J, Patten L, Cervantez D (1994): A catheter-based curative approach to atrial fibrillation in humans. Circulation $\underline{88}, 1-335$

Tajiri J, Hamasaki S, Shimida T, Morita M, Higashi K, Ohishi S, Sato T (1986): Masked thyreoid dysfunction among elderly patients with atrial fibrillation. Jpn Heart J $\underline{27}, 183-190$

Tikoff G, Schmidt AM, Hecht HH (1968): Atrial fibrillation in atrial septal defect. Arch Intern Med 121, 402

Trümper S (2006): Einheitlicher Bewertungsmaßstab (EBM) vom 4. Quartal 2006 auf Grundlage der 115. Sitzung mit Bekanntmachung im Heft 33 Deutsches Ärzteblatt vom 18.8.2006 sowie Erratum in Heft 37 Deutsche Ärzteblatt vom 15.9.2006

Wehrschuetz M, Wehrschuetz E, Schuchlenz H, Schaffler G (2010): Accuracy of MSCT Coronary Angiography with 64 Row CT Scanner-Facing the Facts. Clin Med Insights Cardiol $\underline{4}, 15-22$

Willems S, Weiss C, Ventura R, Ruppel R, Risius T, Hoffmann M, Meinertz T (2000): Catheter ablation of atrial flutter guided by electroanatomic mapping (CARTO): a randomized comparison to the conventional approach. J Cardiovasc Electrophysiol. 11, 1223-1230 
Yang L, Zhang Z, Fan Z, Xu C, Zhao L, Yu W, Yan Z (2009): 64-MDCT coronary angiography of patients with atrial fibrillation: influence of heart rate on image quality and efficacy in evaluation of coronary artery disease. AJR Am J Roentgenol 193, 795-801

Zhang SZ, Hu XH, Zhang QW, Huang WX (2005): Evaluation of computed tomography coronary angiography in patients with a high heart rate using 16-slice spiral computed tomography with 0.37-s gantry rotation time. Eur Radiol $\underline{15}, 1105-1109$ 


\section{Lebenslauf}

Ich, Sebastian Kruse, wurde am 22. Dezember 1982 als Sohn von Dr. med Thomas Kruse, Arzt, und Bettina Kruse, selbstständig, in Aachen geboren.

Ab 1988 besuchte ich die Städtische Gemeinschaftsgrundschule Aachen-Walheim, bevor ich 1993 auf das Indagymnasium Aachen wechselte und dort 2002 meine Allgemeine Hochschulreife erlangte. Von September 2002 bis Juni 2003 absolvierte ich meinen Zivildienst im Seniorenheim Venwegen-Stolberg. Daran schloss sich, während ich auf die Zuteilung eines Studienplatzes im Fach Humanmedizin wartete, die Arbeit als Projektleiter in der Cateringabteilung der Firma mks $\mathrm{GmbH}$ an.

Zum Wintersemester 2005 begann ich schließlich mein Studium der Humanmedizin an der Georg-August-Universität Göttingen und absolvierte nach vier Semestern den „Ersten Abschnitt der Ärztlichen Prüfung“. Während des klinischen Teils meines Studiums sammelte ich praktische Erfahrungen in den Bereichen Innere Medizin, Augenheilkunde, Allgemein- und Viszeralchirurgie, Orthopädie und Intensivmedizin. Nach dem vierten klinischen Semester begann ich meine Promotion mit dem Titel „Diagnostischer Stellenwert der Koronarangiographie mittels Mehrschicht-Computertomographie bei Patienten mit symptomatischem Vorhofflimmern vor Pulmonalvenenablation“ in der Abteilung Kardiologie und Pneumologie der Universitätsmedizin Göttingen unter der Leitung von Prof. Dr. med. M. Zabel und der Betreuung von Dr. med. C. Sohns.

Von 2010 bis 2011 folgte mein Praktisches Jahr, wobei ich das erste Tertial im Fach Neurologie im St. Vinzenz-Krankenhaus Paderborn, das zweite Tertial im Fach Innere Medizin im Luzerner Kantonsspital und das dritte Tertial im Fach Chirurgie im Evangelischen Krankenhaus (Unfallchirurgie) sowie im Pius-Hospital Oldenburg (Allgemein- und Viszeralchirurgie) absolvierte.

Im Herbst 2011 konnte ich schließlich den „Zweiten Abschnitt der Ärztlichen Prüfung ablegen“.

2012 begann ich mit der Weiterbildung zum Allgemeinmediziner in der Medizinischen Klinik des Luisenhospitals Aachen, seit 2015 arbeite ich im Rahmen der Weiterbildung in der hausärztlichen Praxis meines Vaters. 